\title{
Non-Newtonian and viscoplastic models of a vertically-aligned thick liquid film draining due to gravity
}

\author{
Hani Nami Alahmadi ${ }^{1,2, a)}$ and Shailesh Naire ${ }^{1, b}$ \\ 1) School of Computing and Mathematics, Keele University, ST55BG, United Kingdom \\ ${ }^{2)}$ Department of Mathematics, College of Science, Jouf University, Sakaka P.O. Box 2014, \\ Saudi Arabia
}

(Dated: 21 December 2021)

\begin{abstract}
We consider theoretically the two-dimensional flow in a vertically-aligned thick liquid film supported at the top and bottom by wire frames. The film gradually thins as the liquid drains due to gravity. We focus on investigating the influence of non-Newtonian and viscoplastic effects, such as shear thinning and yield stress, on the draining and thinning of the liquid film, important in metallic and polymeric melt films. Lubrication theory is employed to derive coupled equations for a generalised Newtonian liquid describing the evolution of the film's thickness and the extensional flow speed. We use the non-Newtonian (Power-law and Carreau) and viscoplastic (Bingham and Herschel-Bulkley) constitutive laws to describe the flow rheology. Numerical solutions combined with asymptotic solutions predict late-time power-law thinning rate of the middle section of the film. For a Newtonian liquid, a new power law thinning rate of $t^{-2.25}$ is identified. This is in comparison to a thinning rate of $t^{-2}$ predicted for a thin Newtonian liquid film neglecting gravity, suggesting a weak dependence on gravity for the drainage of thicker films. For a non-Newtonian and viscoplastic liquid, varying the power law index and the yield stress influences the time scale of the thinning, but has weak dependence on the late-time thinning rate relative to the Newtonian thinning rate. The shortcomings of the Power-law model are exposed when the shear rate is low and these are resolved using the Carreau model.
\end{abstract}

\section{INTRODUCTION}

The draining, thinning and break-up of liquid films that intertwine a network of gas bubbles are a common occurrence in foams. They are integral to the formation and stability of foams, and in predicting their lifetime ${ }^{1-3}$. The dynamics play a crucial role in a variety of applications, such as in liquid and solid foam networks, relevant in the manufacture of cellulose foams ${ }^{4,5}$, metallic, polymeric and ceramic foams ${ }^{1,6,7}$, the food industry (e.g., bread dough ${ }^{8}$ ), processing in the petro-chemical industry $^{9,10}$, and the biological and life sciences ${ }^{11}$. In a liquid foam network, there are gas bubbles separated by thin liquid lamellae. If one is interested in predicting the lifetime of a foam or its overall stability then, as a starting point, understanding the drainage within the lamella is important.

The process of liquid drainage and thinning of the lamella is well-studied in aqueous Newtonian foams ${ }^{3}$ where surfactants are required to stabilize foams. In pure molten metal and polymeric foams, however, the drainage and thinning of the lamella is rapid and rupture can happen in the order of milliseconds. Surfactants are not available to affect the surface tension of metallic foams. Therefore, particles are often added to metallic foams to increase the effective liquid viscosity and to slow down the drainage, thinning and rupture time ${ }^{1,12}$. Such foams display non-Newtonian, such as shear-thinning, viscoelastic and viscoplastic, such as a yield stress, be-

\footnotetext{
a)hnalahmadi@ju.edu.sa

b) s.naire@keele.ac.uk; Corresponding author
}

haviour. Foams made from non-Newtonian and viscoelastic solutions have attracted much less attention so far in comparison to Newtonian foams and there are few studies on their drainage. Safouane et al. ${ }^{13}$ have performed forced drainage experiments in nonNewtonian foam solutions displaying shear-thinning behaviour. Their results showed that the dynamics of liquid drainage in a foam with a shear-thinning liquid solution is identical to the drainage of foam having a Newtonian liquid if an effective viscosity based on an estimated shear rate within the foam is used. They also demonstrated that foams made of aqueous solutions of a polymer drain faster than foams made with Newtonian solutions of the same bulk viscosity ${ }^{13,14}$. They attributed this behaviour to the viscoelastic properties of the polymer. Indeed, experimental investigation by Koponen ${ }^{4}$ on drainage of fibre-laden cellulose foams have shown that the general drainage behaviour is similar to that of a pure foam model ${ }^{5}$, however, the time scale of drainage given by the model was almost an order of magnitude longer than in the experiments. The authors concluded that the time scales could be matched by using an appropriate effective viscosity, similar to Safouane et al. ${ }^{13}$, but could not rigorously justify this method by the known properties of the system. A quantitative analysis of the drainage of nonNewtonian and viscoelastic films and foams is therefore important from both a practical and theoretical point of view.

The classical experimental investigation by Mysels, Shinoda \& Frankel $^{15}$ using soap films attached to wire frames gave the first comprehensive description of the draining and thinning of soap films. Subsequently, this has spurred several experimental and theoretical studies investigating the thinning and drainage of liquid 
films using the model systems for Newtonian liquids under isothermal conditions. These include a liquid film supported within a wire frame or between two rigid supports ${ }^{15,16}$, or a liquid film partially drawn out of a bath of liquid ${ }^{17-21}$. These two configurations mimic the fluid dynamics associated with the draining of a lamella into a Plateau border. The latter configuration also allows investigation of the speed at which the wire frame is drawn out of the bath, and its influence on the stability of the draining film ${ }^{17}$.

The draining and thinning of free liquid films have been widely studied for a surfactant-free ${ }^{22-25}$ system using a purely extensional flow framework, and for a surfactantstabilized system using a combination of extensional and shear flow ${ }^{16,18-21,23,24,26}$. Particularly relevant to this work are the following studies. Breward ${ }^{23}$ and Breward \& Howell ${ }^{24}$ have developed and analysed models describing surfactant-free and surfactant-stabilized drainage of a foam lamella. For the capillary number, $C a=\mu^{\star} U^{\star} / \gamma^{\star}=O(\epsilon)$ (where $\mu^{\star}$ and $\gamma^{\star}$ are the liquid viscosity and surface tension, respectively, $U^{\star}$ is a characteristic speed and $\epsilon \ll 1$ is the film's aspect ratio), they performed an asymptotic decomposition of the liquid domain into a capillary-static Plateau border, a time-dependent thinning film and a quasi-steady transition region between the two. They use matched asymptotic analysis to describe the evolution of each region, which is then used to derive the thinning rates with and without surfactant. They determined that the lamella drains or the film thins as $t^{-2}$, where $t$ is the time, for a surfactant-free film. They found that surfactants can reduce the draining of the lamella and greatly increase its lifetime. Brush \& Davis ${ }^{25}$ derive the thinning rate of a surfactant-free lamella in a gas-liquid foam using matched asymptotic analysis, in the spirit of Breward ${ }^{23}$ and Breward \& Howell ${ }^{24}$. Two limiting cases were identified at small capillary number: a semi-arid foam having $O(1)$ liquid fraction and an arid foam in which the liquid fraction is small. They found that the long-time lamellar thinning rates in both cases followed a $t^{-2}$ power-law behaviour; the dynamics and rupture times were different for both foam types.

The influence of gravity on the drainage of surfactantstabilised aqueous films has been widely studied, in particular its influence on the drainage, thinning and rupture of the lamella ${ }^{16,18-21}$. However, the gravity-driven drainage of surfactant-free films is relatively understudied, generally neglected under the assumption that the film is thin. To the best of our knowledge, the only study to have examined the role of gravity in the drainage and thinning of flow from the lamella into the Plateau border is by Davis et al. ${ }^{27}$. They combine numerical simulations and asymptotic analysis (based on the domain decomposition procedure ${ }^{24,25}$ ) to demonstrate how gravitational effects strongly modify the shape of the Plateau border interfaces and enhance the drainage flow in the liquid films. The lamella thins non-uniformly with exponential decay of the minimum film thickness, which is signifi- cantly faster than the $t^{-2}$ power-law thinning predicted when gravitational effects are negligible ${ }^{23-25}$. Motivated by this study, we focus on the scenario when the liquid film is sufficiently thick so that the liquid flow driven by gravity is non-negligible, and could significantly influence its draining and thinning.

Even fewer models have considered the influence of non-Newtonian rheology, such as shear-thinning and viscoplastic behaviour. Brush \& Roper ${ }^{28}$ extend their twodimensional small capillary number matched asymptotic analysis for Newtonian liquids ${ }^{25}$ to determine the thinning rates of thin liquid films in surfactant-free, nonNewtonian gas-liquid foams. The liquid viscosity is modelled as a power-law function of the shear rate and by the Ellis law ${ }^{29}$. They observed that the Ellis model is more realistic than the Power-law model at a low shear rate, where the viscosity is well behaved in the Ellis model while it diverges to infinity in the Power-law model. They observed the thinning rate to be $t^{-2}$ for both models, which is the same as that for a Newtonian liquid. They reasoned that the non-Newtonian behaviour influences the time to rupture without affecting the thinning rate. Although the work undertaken here is in the spirit of the study by Brush \& Roper ${ }^{28}$, we derive the evolution equations in an uncomplicated way, and assume that the liquid film is relatively thick so that the effect of gravity is included.

Based on the above considerations, the goal of this paper is twofold: firstly, to develop a theoretical framework for the draining of a vertically-aligned free thick liquid film, incorporating gravity, extensional viscous, surface tension and a shear-rate dependent viscosity for a generalized Newtinian liquid. Secondly, to investigate the influence of non-Newtonian effects, such as shear thinning, and viscoplastic effects, such as yield stress, on the draining and thinning of the liquid film. The plan of the paper is as follows. In $\S I I$, we focus on the distinguished limit balancing the extensional viscous stresses and gravity to derive the master equations for a generalised Newtonian liquid describing the time-evolution of the film's free surface and the extensional flow speed. In $\S I I I$, we explore the solutions when the rescaled capillary number, $\mathrm{Ca} / \epsilon=O(1)$ or larger. This is done first for a Newtonian liquid and then for a non-Newtinian liquid using the Power-law and Carreau models to describe the shear-thinning and thickening behaviour, and the Herschel-Bulkley and Bingham model to describe the weakly yielding behaviour. In $\S \mathrm{IV}$, we provide concluding remarks and outline future work.

\section{MODEL FORMULATION}

We consider the two-dimensional flow due to the draining of a liquid in a vertically-aligned film with two free surfaces and suspended between two horizontal solid frames, as shown in Fig. 1. This configuration assumes the pre-existence of a stable initial liquid film of specified 
height and thickness. In experiments, the film is drawn out of a bath of liquid and whether a film of specified height and thickness can be achieved would depend on the the speed at which it is drawn out ${ }^{17}$. This speed also significantly influences the stability of the draining film ${ }^{17}$. Here we assume that the film is drawn out sufficiently quickly for a stable initial film profile to exist.

The configuration shown in Fig. 1 mimics the thinning of the lamella and is a simple idealization of the physical situation. However, the supported ends are not a true replication of the Plateau borders in liquid foam films. One would need to consider lifting the wire frame completely out of a bath of liquid resulting in a liquid film that connects onto the bath at its lower end (for example, see Champougny et $a l^{17}$ and Naire et $\left.a l^{18-21}\right)$. The bottom end of the film connecting onto the bath's surface is representative of the Plateau border region. Nonetheless, the current theoretical framework is still applicable to this configuration, except that one needs to apply appropriate boundary conditions for the film to match onto the bath. As we will see below, this is much simpler with the configuration shown in Fig. 1.

The initial liquid film is sufficiently thick for gravity to play a significant role in its drainage. The flow evolves due to the effects of gravity, viscous forces and surface tension causing the liquid in the film to drain downwards in the direction of gravity and resulting in the thinning of the film. The liquid is assumed to be an incompressible and generalised Newtonian liquid with a shear ratedependent viscosity. We also assume isothermal conditions, so the liquid properties are independent of variations in temperature. Figure 1 shows a schematic of the

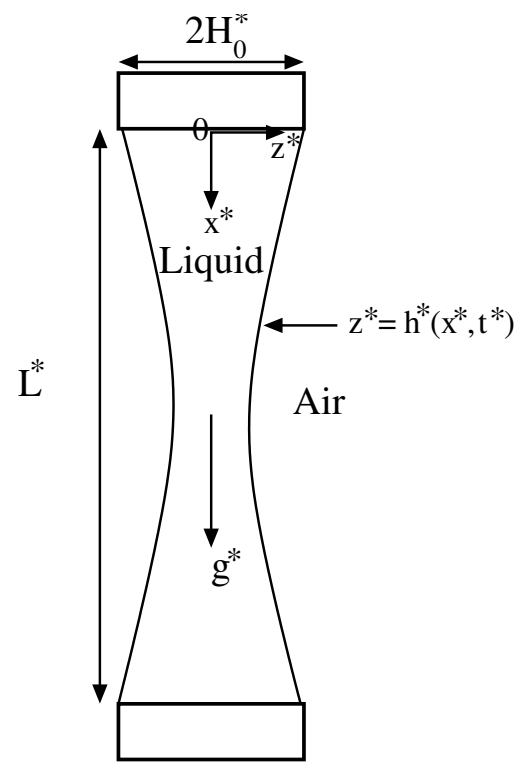

FIG. 1: Schematic of a vertically-aligned two-dimensional free liquid film draining under gravity between two rigid frames.

geometry. We consider a two-dimensional Cartesian co- ordinate system $\left(x^{\star}, z^{\star}\right)$ with the $x^{\star}$-axis in the vertical direction pointing downwards in the direction of the film length and the $z^{\star}$-axis in the horizontal direction along the film's thickness. The horizontal frames are separated by a distance $L^{\star}$ and are of width $2 H_{0}^{\star}$. Gravity acts vertically downwards. We assume symmetry about the film's centre line at $z^{\star}=0$. The two free surfaces of the film are represented by $z^{\star}= \pm h^{\star}(x, t)$. Assuming left-right symmetry, we only consider half of the film between $z^{\star}=0$ and $z^{\star}=h^{\star}(x, t)$. The superscript ${ }^{\star}$ refers to dimensional quantities.

\section{A. Governing equations}

The flow is described by the Navier-Stokes equations. The density $\rho^{\star}$ is assumed constant (due to the incompressibility assumption), so the continuity equation reduces to

$$
u_{x^{\star}}^{\star}+w_{z^{\star}}^{\star}=0 .
$$

In the above, $\mathbf{v}^{\star}=\left(u^{\star}, w^{\star}\right)$ are the flow speeds in the $x^{\star}$ and $z^{\star}$ directions, respectively, and the subscript denotes differentiation with respect to the subscript variable. The momentum equations can be written as:

$$
\begin{aligned}
& \rho^{\star}\left(u_{t^{\star}}^{\star}+u^{\star} u_{x^{\star}}^{\star}+w^{\star} u_{z^{\star}}^{\star}\right)=-p_{x^{\star}}^{\star}+\tau_{x^{\star}}^{\star} x x+\tau_{z^{\star}}^{\star} x z+\rho^{\star} g^{\star}, \\
& \rho^{\star}\left(w_{t^{\star}}^{\star}+u^{\star} w_{x^{\star}}^{\star}+w^{\star} w_{z^{\star}}^{\star}\right)=-p_{z^{\star}}^{\star}+\tau_{x^{\star}}^{\star} x z+\tau_{z^{\star}}^{\star} z z,
\end{aligned}
$$

where $p^{\star}$ is the liquid pressure, $\tau^{\star} x x$ and $\tau^{\star} z z$ are the extensional viscous stresses in the $x^{\star}$ and $z^{\star}$ directions, respectively, $\tau^{\star} x z$ is the viscous shear stress and and $g^{\star}$ is the acceleration due to the gravity.

We use a constitutive law for a generalised Newtonian liquid relating the viscous stress $\tau^{\star}$ and the shear rate $\dot{\gamma^{\star}}$ of the form:

$$
\tau^{\star}=\mu^{\star}\left(\dot{\gamma^{\star}}\right) \dot{\gamma}^{\star},
$$

where $\mu^{\star}\left(\gamma^{\star}\right)$ is the shear rate-dependent viscosity, $\dot{\gamma^{\star}}=\left[\frac{1}{2} \operatorname{Trace}\left(\dot{\gamma^{\star}} \cdot \dot{\gamma}^{\star}\right)\right]^{1 / 2}=\left[2\left(u_{x^{\star}}^{\star^{2}}+w_{z^{\star}}^{\star^{2}}\right)+\left(u_{z^{\star}}^{\star}+w_{x^{\star}}^{\star}\right)^{2}\right]^{1 / 2}$, is the second invariant of the shear rate tensor, and

$$
\begin{aligned}
\tau^{\star} & =\left(\begin{array}{ll}
\tau^{\star} x x & \tau^{\star} x z \\
\tau^{\star} x z & \tau^{\star} z z
\end{array}\right), \\
\dot{\gamma^{\star}} & =\left(\begin{array}{ll}
\gamma^{\star} x x & \gamma^{\star} x z \\
\gamma^{\star} x z & \gamma^{\star} z z
\end{array}\right)=\left(\begin{array}{cc}
2 u_{x^{\star}}^{\star} & u_{z^{\star}}^{\star}+w_{x^{\star}}^{\star} \\
u_{z^{\star}}+w_{x^{\star}}^{\star} & 2 w_{z^{\star}}^{\star}
\end{array}\right),
\end{aligned}
$$

\section{B. Boundary conditions}

Symmetry along the center line $z^{\star}=0$ is imposed through the boundary conditions:

$$
w^{\star}=u_{z^{\star}}^{\star}=\tau^{\star} x z=0, \text { at } z^{\star}=0 .
$$


At the free surface, $z^{\star}=h^{\star}\left(x^{\star}, t^{\star}\right)$, we have the stress boundary conditions normal and tangential to the free surface. The normal stress boundary condition balances the jump in the total normal stress (between the outside air and the liquid) with the product of the surface tension times the curvature of the free surface,

$-p^{\star}+\frac{1}{1+h_{x^{\star}}^{\star^{2}}}\left[h_{x^{\star}}^{\star^{2} \tau^{\star} x x}-2 h_{x^{\star}}^{\star} \tau^{\star} x z+\tau^{\star} z z\right]=\frac{\gamma^{\star} h_{x^{\star} x^{\star}}^{\star}}{\left(1+h_{x^{\star}}^{\star^{2}}\right)^{\frac{3}{2}}}$,

where $\gamma^{\star}$ is the surface tension (assumed constant) and $h_{x^{\star} x^{\star}}^{\star} /\left(1+h_{x^{\star}}^{\star^{2}}\right)^{\frac{3}{2}}$ is the surface curvature. Without loss of generality, we take the atmospheric pressure to be zero, therefore, the liquid pressure $p^{\star}$ is relative to the atmospheric pressure. The tangential stress boundary condition imposes continuity of stress at the free surface. Assuming that the stress applied in the outside air phase is zero, this can be written as:

$$
\left(1-h_{x^{\star}}^{\star^{2}}\right) \tau^{\star} x z+h_{x}\left(\tau^{\star} z z-\tau^{\star} x x\right)=0 .
$$

Finally, the kinematic boundary condition at the free surface is given by

$$
h_{t^{\star}}^{\star}=w^{\star}-u^{\star} h_{x^{\star}}^{\star}, \text { at } z^{\star}=h^{\star}\left(x^{\star}, t^{\star}\right) .
$$

At the top and bottom boundary, $x^{\star}=0, L^{\star}$, respectively, the film is pinned to the end of the frame and we impose no slip,

$$
h^{\star}=H_{0}^{\star} \text { and } \mathbf{v}^{\star}=0, \text { at } x^{\star}=0, L^{\star} .
$$

Using Eq. (1), and applying Leibniz's rule, one can re-write the kinematic boundary condition, Eq. (8), as

$$
h_{t^{\star}}^{\star}+Q_{x^{\star}}^{\star}=0, \quad Q^{\star}=\int_{0}^{h^{\star}} u^{\star}\left(x^{\star}, z^{\star}, t^{\star}\right) d z^{\star},
$$

where $Q^{\star}\left(x^{\star}, t^{\star}\right)$ is the liquid flux at any location $x^{\star}$ along the length of the film. Eq. (10) represents the evolution of the film thickness, $h^{\star}\left(x^{\star}, t^{\star}\right)$.

\section{Nondimensionalization of the governing equations and boundary conditions}

The governing equations and boundary conditions are nondimensionalised using

$$
\begin{aligned}
& x^{\star}=L^{\star} x,\left(z^{\star}, h^{\star}\right)=H_{0}^{\star}(z, h), u^{\star}=U^{\star} u, w^{\star}=W^{\star} w, \\
& \left(p^{\star}, \tau^{\star} x x, \tau^{\star} z z, \tau^{\star} x z\right)=\left(P^{\star} p, T^{\star} \tau^{x x}, T^{\star} \tau^{z z}, \frac{T^{\star}}{\epsilon} \tau^{x z}\right), \\
& \left(\gamma^{\star} x x, \gamma^{\star} z z, \gamma^{\star} x z\right)=\mu_{0}^{\star}\left(\frac{U^{\star}}{L^{\star}} \gamma^{x x}, \frac{U^{\star}}{L^{\star}} \gamma^{z z}, \frac{U^{\star}}{\epsilon L^{\star}} \gamma^{x z}\right), \\
& t^{\star}=\frac{L^{\star}}{U^{\star}} t, Q^{\star}=U^{\star} H_{0}^{\star} Q,
\end{aligned}
$$

where $\mu_{0}^{\star}$ is a reference viscosity of the liquid and $U^{\star}$, $W^{\star}, P^{\star}$ and $T^{\star}$ are characteristic speeds, pressure and stress, respectively. The ratio of the two length scales is denoted by $\epsilon=\frac{H_{0}^{\star}}{L^{\star}}$, which is typically much less than one. We are interested in deriving the thin film equations in the asymptotic limit $\epsilon \rightarrow 0$.

We focus on the scenario where the flow is primarily extensional (or plug flow) and there is a balance between extensional viscous stresses and gravity. In this case, the downward pressure gradient $\left(p_{x^{\star}}^{\star}\right)$ is comparable to the gradient of the extensional viscous stress $\left(\tau_{x^{*}}^{x^{*}}\right)$ in Eq. (2a). So, $p_{x^{\star}}^{\star} \sim \tau_{x^{\star}}^{\star} x x$ which implies that the stress scale $T^{\star}=P^{\star}$. We also demand that in Eq. (2a) the downward pressure gradient $\left(p_{x^{\star}}^{\star}\right)$ is comparable to the force due to gravity $\left(\rho^{\star} g^{\star}\right)$, hence the characteristic pressure scale $P^{\star}=\rho^{\star} g^{\star} L^{\star}$, so the characteristic stress scale $T^{\star}=\rho^{\star} g^{\star} L^{\star}$. The extensional flow speed $U^{\star}$ will be determined later based on a distinguished limit. Eq. (1), implies $u_{x^{\star}}^{\star} \sim w_{z^{\star}}^{\star}$ which gives $W^{\star}=\epsilon U^{\star}$. Table I provides the values of the dimensional quantities based on a viscous silicon oil as a representative Newtonian liquid.

\begin{tabular}{|c|c|}
\hline Dimensional quantities & Values \\
\hline density, $\rho^{\star}$ & $10^{3} \mathrm{~kg} / \mathrm{m}^{3}$ \\
viscosity, $\mu_{0}^{\star}$ & $10 \mathrm{~Pa} \mathrm{~s}$ \\
surface tension, $\sigma^{\star}$ & $42 \mathrm{mN} / \mathrm{m}$ \\
length, $L^{\star}$ & $10^{-2} \mathrm{~m}$ \\
width, $H_{0}^{\star}$ & $50 \mu \mathrm{m}$ \\
characteristic speed, $U^{\star}=\frac{\rho^{\star} g^{\star} L^{\star^{2}}}{\mu_{0}^{\star}}$ & $0.1 \mathrm{~m} / \mathrm{s}$ \\
characteristic pressure, $p^{\star}=\rho^{\star} g^{\star} L^{\star}$ & $10^{3} \mathrm{~N} / \mathrm{m}^{2}$ \\
characteristic time, $t^{\star}=\frac{L^{\star}}{U^{\star}}$ & $0.1 \mathrm{~s}$ \\
\hline
\end{tabular}

TABLE I: Characteristic values of the dimensional quantities, assuming that the liquid is silicon oil.

Substituting Eq. (11) into the governing equations and boundary conditions gives the following nondimensional- 
ized system:

$$
\begin{aligned}
& u_{x}+w_{z}=0, \\
& \epsilon^{2} \operatorname{Re}\left(u_{t}+u u_{x}+w u_{z}\right)=-\epsilon^{2} p_{x}+\epsilon^{2} \tau_{x}^{x x}+\tau_{z}^{x z}+ \\
& \epsilon^{2} B, \\
& \epsilon^{2} R e\left(w_{t}+u w_{x}+w w_{z}\right)=-p_{z}+\tau_{x}^{x z}+\tau_{z}^{z z}, \\
& \left(\begin{array}{ll}
\tau^{x x} \tau^{x z} \\
\tau^{x z} \tau^{z z}
\end{array}\right)=\mu(\dot{\gamma})\left(\begin{array}{cc}
2 u_{x} & u_{z}+\epsilon^{2} w_{x} \\
u_{z}+\epsilon^{2} w_{x} & 2 w_{z}
\end{array}\right),(12 \mathrm{~b}) \\
& w=u_{z}=\tau^{x z}=0, \text { at } z=0, \\
& \frac{\epsilon}{\hat{C a}} \frac{h_{x x}\left(1+\epsilon^{2} h_{x}^{2}\right)^{\frac{3}{2}}}{(12 \mathrm{c})}-p+ \\
& \frac{1}{1+\epsilon^{2} h_{x}^{2}}\left[\epsilon^{2} h_{x}^{2} \tau^{x x}-2 h_{x} \tau^{x z}+\tau^{z z}\right], \text { at } z=h(x, t), \\
& \left(1-\epsilon^{2} h_{x}^{2}\right) \tau^{x z}+\epsilon^{2} h_{x}\left(\tau^{z z}-\tau^{x x}\right)=0, \text { at } z=h(x, t), \\
& h_{t}+Q_{x}=0, \quad Q=\int_{0}^{h} u(x, z, t) d z, \\
& h=1, u=w=0, \text { at } x=0,1 .
\end{aligned}
$$

The dimensionless form of the function $\mu(\dot{\gamma})$ depends on the constitutive law used, e.g., for a Newtonian liquid, $\mu(\dot{\gamma})=1$.

In the above, the dimensionless number $B=\frac{\rho^{\star} g^{\star} L^{\star^{2}}}{\mu_{0}^{\star} U^{\star}}$ compares gravity and extensional viscous forces, $R e=$ $\frac{\rho^{\star} U^{\star^{2}} / L^{\star}}{\mu_{0}^{\star} U^{\star} / L^{\star^{2}}}$ is the Reynolds number (compares inertial and extensional viscous forces) and $\hat{C a}=\frac{\mu_{0}^{\star} U^{\star}}{\gamma^{\star}}$ is the capillary number (compares extensional viscous and surface tension forces). There are 2 distinguished limits to be considered here based mainly on the order of magnitude of $B$ and $R e$. We also note a third distinguished limit which is not the focus of this work.

(i) Balancing extensional viscous forces and gravity, $B \sim 1^{16,17}$. This gives a characteristic speed $U^{\star} \sim$ $\frac{\rho^{\star} g^{\star} L^{\star} 2}{\mu_{0}^{\star}} \sim 0.1 \mathrm{~m} / \mathrm{s}$. The inertial forces are smaller in comparison, with corresponding $R e \sim 0.1$.

(ii) Balancing extensional viscous forces and inertia, $R e \sim 1^{22}$. This gives a characteristic speed $U^{\star} \sim$ $\frac{\mu_{0}^{\star}}{\rho^{\star} L^{\star}} \sim 1 \mathrm{~m} / \mathrm{s}$. The force due to gravity is smaller in comparison, with corresponding $B \sim 0.1$.

(iii) If $B \sim 1 / \epsilon^{2}$, i.e., corresponding $U^{\star} \sim \epsilon^{2}$, then the leading order balance is between viscous shear forces and gravity (see, for example, Naire et al. $\left.{ }^{18-21}\right)$.

Our work focusses on the balance between extensional viscous forces and gravity, hence we set $B=1$, which sets the characteristic speed $U^{\star}=\frac{\rho^{\star} g^{\star} L^{\star} 2}{\mu_{0}^{\star}} \sim 0.1 \mathrm{~m} / \mathrm{s}$. The corresponding Reynolds number $R e=0.1$. We will see later on, that surface tension effects will be important over smaller lengthscales, so in anticipation of this we define a rescaled capillary number, $C a=\frac{\mu_{0}^{\star} U^{\star}}{\epsilon \gamma^{\star}}=\hat{C} a / \epsilon$, $\hat{C} a=O(1)$, and retain the surface tension term at leading order. Estimates of the dimensionless parameters are provided in Table II.

\begin{tabular}{|c|c|}
\hline Dimensional quantities & Values \\
\hline$\epsilon=H_{0}^{\star} / L^{\star}$ & $10^{-2}$ \\
$B=\frac{\rho^{\star} g^{\star} L^{\star} 2}{\mu_{0}^{\star} U^{\star}}$ & 1 \\
$R e=\frac{\rho^{\star} U^{\star} L^{\star}}{\mu_{0}^{\star} U^{\star}}$ & 0.1 \\
$\hat{C} a=\frac{\mu_{0}^{\star} U^{\star}}{\gamma^{\star}}$ & 25 \\
$C a=\frac{\hat{C} a}{\epsilon}$ & $2.5 \times 10^{3}$ \\
\hline
\end{tabular}

TABLE II: Estimates of the dimensionless parameters.

\section{The master PDEs}

We use the lubrication approximation exploiting the fact that $\epsilon=\frac{H_{0}^{\star}}{L^{\star}} \ll 1$ and expand each of the unknowns variables $\left(u, w, p, \tau^{x x}, \tau^{z z}, \tau^{x z}, h\right)$ as a power series in $\epsilon^{2}$ of the form:

$$
\begin{aligned}
& \left(u, w, p, \tau^{x x}, \tau^{z z}, \tau^{x z}, h\right)=\left(u, w, p, \tau^{x x}, \tau^{z z}, \tau^{x z}, h\right)_{0}(x, z, t) \\
& +\epsilon^{2}\left(u, w, p, \tau^{x x}, \tau^{z z}, \tau^{x z}, h\right)_{1}(x, z, t)+O\left(\epsilon^{4}\right) .
\end{aligned}
$$

Substituting this in Eq. (12) we can sequentially solve for the $O(1)$ and $O\left(\epsilon^{2}\right)$ quantities, using which the master system of PDEs and boundary conditions for the evolution of the film's free surface $h_{0}(x, t)$ and the extensional flow speed $u_{0}(x, t)$ can be derived at leading order. The details of the derivation are provided in Appendix A. The master system of PDEs and boundary conditions are given by: (for brevity, we drop the subscript 0 )

$$
\begin{aligned}
h_{t}+Q_{x} & =0 \\
Q= & u h+\epsilon^{2} \frac{h^{3}}{3}\left[\frac { 1 } { \mu ( | u _ { x } | ) } \left(4\left(\mu\left(\left|u_{x}\right|\right) u_{x}\right)_{x}\right.\right. \\
& \left.\left.+\frac{1}{C a} h_{x x x}+1-\operatorname{Re}\left(u_{t}+u u_{x}\right)\right)-u_{x x}\right],
\end{aligned}
$$

$\operatorname{Re} h\left(u_{t}+u u_{x}\right)-4\left(h \mu\left(\left|u_{x}\right|\right) u_{x}\right)_{x}-h\left[\frac{1}{C a} h_{x x x}+1\right]=0$,

$h(0, t)=h(1, t)=1, h_{x x x}(0, t)=h_{x x x}(1, t)=-C a$,

$u(0, t)=u(1, t)=0$. 
Eq. (14d) provide the six boundary conditions required in total for Eqs. $(14(a-c))$ which correspond physically to the film being pinned at the top and bottom (first two boundary conditions in Eq. (14d)), and no flux out of the rigid wire supports, so $Q=0$, (represented by the last four boundary conditions in Eq. (14d)). As a consequence of this, both $u$ and $u_{x}$ are forced to be zero near the ends and the film evolves to quasi-static shapes there. In Eq. (14b), the contribution from the shear flow (second term on the right-hand side) is $O\left(\epsilon^{2}\right)$ smaller than the extensional component (first term on the right-hand side). Hence, to leading order in $\epsilon$, the evolution of the film thickness given by Eq. (14a) is hyperbolic. We include the $O\left(\epsilon^{2}\right)$ terms as a regularization for the boundary conditions in Eq. (14d) to be satisfied. Eq. (14) are parametrised by the constitutive relationship for $\mu\left(\left|u_{x}\right|\right)$, the capillary number $C a$ and the Reynold's number, $R e$. We seek solutions of Eq. (14) for $C a \gg 1$, which corresponds to much weaker surface tension in comparison to gravity, and $R e \ll 1$.

Similar equations for a generalised Newtonian liquid have been derived by Brush \& Roper $^{28}$, without the $O\left(\epsilon^{2}\right)$ regularisation terms, and not including the effect of gravity. In the case of a Newtonian liquid, the equations and boundary conditions are the same as those derived by Schwartz \& Roy ${ }^{16}$, except that we do not include surfactants or surfactant-related effects here. Similar evolution equations have also been derived focussing on particular balance of physical effects, e.g., Erneux \& Davis ${ }^{22}$ (extensional flow balancing inertia, not including gravity), Champougny et al. ${ }^{17}$ (extensional flow balancing gravity and van der Waals forces) and Breward ${ }^{23,24}$, Brush \& Davis $^{25}$ (balancing extensional flow with surface tension effects, not including gravity) and Davis et al. ${ }^{27}$ (balancing extensional flow with surface tension effects, including gravity).

We consider different functional forms of the constitutive law for $\mu\left(\left|u_{x}\right|\right)$ in Eq. (14) corresponding to liquids displaying generalised Newtonian (represented by Powerlaw and Carreau models) and viscoplastic behaviour (represented by the Herschel-Bulkley model). These can be written as:

$$
\begin{aligned}
\mu\left(\left|u_{x}\right|\right)=K\left|u_{x}\right|^{n-1}, \quad \text { (Power-law) } & \\
\mu\left(\left|u_{x}\right|\right)= & K\left|u_{x}\right|^{n-1}+\frac{\tau_{p}}{\left|u_{x}\right|}, \text { if } \tau^{x x}>\tau_{p}, \\
& u_{x}=0, \text { otherwise, } \quad \text { (Herschel-Bulkley) }
\end{aligned}
$$

$$
\mu\left(\left|u_{x}\right|\right)=\mu_{\infty}+\left(1-\mu_{\infty}\right)\left[1+\left(\lambda\left|u_{x}\right|\right)^{2}\right]^{(n-1) / 2},
$$$$
\text { (Carreau) }
$$

where $K=2^{n-1}\left(K^{\star} / \mu_{0}^{\star}\right)\left(U^{\star} / L^{\star}\right)^{n-1}$, is a dimensionless liquid consistency index, $n$ is the power-law index (for $n<1$, the fluid is shear thinning; $n>1$ the fluid is shear thickening; $n=1$ is the Newtonian case), $\tau_{p}=\frac{\tau_{p}^{\star}}{\mu_{0}^{\star} U^{\star} / L^{\star}}$ is the Bingham number which compares the liquid yield stress to the extensional viscous stress, $\mu_{\infty}^{\star}=\mu_{\infty}^{\star} / \mu_{0}^{\star}$ is the viscosity in the limit of large shear rate, and $\lambda=\lambda^{\star} L^{\star} / U^{\star}$ is a relaxation time. Characteristic values of these parameters are based on those reported for polymeric liquids, Polystyrene, Aluminium soap and Hydroxylethycellulose, in Table 1 in Myers ${ }^{29}$. For Polystyrene, $n=0.39$ (Power-law model), $K^{\star}=3.5 \times 10^{5} \mathrm{~Pa} \mathrm{~s}{ }^{n}$, $n=0.4$ (Carreau model), $\mu_{1}^{\star}=4 \times 10^{6} \mathrm{~Pa} \mathrm{~s}$ and $\lambda^{\star}=46.4 \mathrm{~s}^{-1}$. This corresponds to $K=12$ and $\lambda=10^{-3}$, assuming the reference viscosity $\mu_{0}^{\star}=\mu_{1}^{\star}$. For Aluminium soap, $n=0.2$ (Power-law model), $K^{\star}=68.07$ $\mathrm{Pa} \mathrm{s}^{n}, n=0.2$ (Carreau model), $\mu_{1}^{\star}=89.6 \mathrm{~Pa} \mathrm{~s}$ and $\lambda^{\star}=1.41 \mathrm{~s}^{-1}$. This corresponds to $K=0.7$ and $\lambda=1$, assuming the reference viscosity $\mu_{0}^{\star}=\mu_{1}^{\star}$. For Hydroxylethycellulose, $n \approx 0.51$ (Power-law model), $K^{\star}=0.84$ $\mathrm{Pa} \mathrm{s}{ }^{n}, n \approx 0.51$ (Carreau model), $\mu_{1}^{\star}=0.22 \mathrm{~Pa} \mathrm{~s}$ and $\lambda^{\star} \approx 0.067 \mathrm{~s}^{-1}$. This corresponds to $K \approx 0.1$ and $\lambda \approx 30$, assuming the reference viscosity $\mu_{0}^{\star}=\mu_{1}^{\star}$.

We note that $\mu\left(\left|u_{x}\right|\right)$ in Eqs. $(15(b, c))$ has a singularity when $u_{x}=0$ for $n<1$, which occurs near the top and bottom of the draining film, and where the flow speed attains its maximum value. In practise, we relieve this singularity by regularising the Power-law and Herschel Bulkley models by adding a sufficiently small positive number, $\delta$, to $u_{x}$, namely,

$\mu\left(\left|u_{x}\right|\right)=K\left[{\sqrt{u_{x}^{2}+\delta^{2}}}^{n-1}, \quad\right.$ (regularised Power-law),

$\mu\left(\left|u_{x}\right|\right)=K\left[{\sqrt{u_{x}^{2}+\delta^{2}}}^{n-1}+\frac{\tau_{p}}{\sqrt{u_{x}^{2}+\delta^{2}}}\right.$.

(regularised Herschel-Bulkley)

The effect of regularization on the Herschel-Bulkley model is that at low shear rates, the fluid becomes weakly yielding when $\tau^{x x}<\tau_{p}{ }^{30}$.

\section{RESULTS}

In the numerical results to follow we mainly focus on investigating the influence of the capillary number $C a$, the consistency index $K$, the Power-law index $n$ and the yield stress $\tau_{p}$, on the evolution of the film thickness $h(x, t)$ and extensional flow speed $u(x, t)$. We also investigate the influence of the more realistic Carreau model and the corresponding index $n$. The parameter range investigated are based on the estimates provided above. $R e \ll 1$ has no significant influence on the evolution of the film and the extensional speed, hence for all the results to follow we choose $R e=0$. The regularization parameter, $\delta$, is chosen based on solving a simplified version of the governing equations in which $h=1$ (which is chosen to be the initial condition for $h$ ). For this case, Eq. (14c) with $R e=0$ has an analytical solution for $u$ given by Eq. (17) using the non-regularized Power-law constitutive model (Eq. (15a). We then numerically solve Eq. (14c) with $h=1$ and $R e=0$ using the regularised Power-law model (Eq. (16a), and choose the value of the 
regularisation parameter $\delta$ so that the error between the numerical and analytical solutions is less than a specified tolerance for a given set of parameter values. This value of $\delta$ is then kept fixed throughout the time evolution. The initial condition is $h(x, 0)=1$, and the corresponding initial condition for the extensional flow speed $u(x, 0)$ obtained by solving Eq. (14c) for $h=1$ and $R e=0$, is given by

$$
\begin{aligned}
u(x, 0)= & \frac{4 K n}{n+1}\left[\left[\frac{(1-L)}{4 K}\right]^{\frac{n+1}{n}}-\left[\frac{(x-L)}{4 K}\right]^{\frac{n+1}{n}}\right] \\
& (\text { for } L \leq x \leq 1), \\
u(x, 0)= & \frac{4 K n}{n+1}\left[\left[\frac{1}{4 K} L\right]^{\frac{n+1}{n}}-\left[\frac{1}{4 K}(L-x)\right]^{\frac{n+1}{n}}\right] \\
& (\text { for } 0 \leq x \leq L),
\end{aligned}
$$

with $L=1 / 2$, for the value of $n$ chosen. In the case when $n=1$, the analytical solution is $u(x, 0)=x(1-x) / 8$.

We discretize Eq. (14) on a uniform mesh using second-order finite difference method for the spatial derivatives, but keep the time derivative continuous. The resulting system of differential-algebraic equations are solved using the method of lines ${ }^{31}$. We use the implicit solver ode15i in MATLAB (MATLAB 6.1, The MathWorks Inc., Natick, MA, 2000) for our numerical simulations. This solves a system of equations of the form $f\left(t, y, y^{\prime}\right)=0$, for the dependent variable $y$. The unknowns $h_{i}$ and $u_{i}$ are numbered according to $y=\left(h_{1}, u_{1}, h_{2}, u_{2}, \ldots, h_{N+1}, u_{N+1}\right)$. This enables the Jacobian matrix to have a much smaller bandwidth which accelerates the computations, compared to a numbering system, for example, $y=$ $\left(h_{1}, h_{2}, \ldots, h_{N+1}, u_{1}, u_{2}, \ldots, u_{N+1}\right)$, which has a much bigger bandwidth. In all the results to follow we choose the mesh size $\Delta x=5 \times 10^{-4}$ for accuracy and convergence of the solutions.

We first consider the case of a Newtonian liquid with $n=1$. Fig. $2(a, b, c)$ show the evolution of $h(x, t)(h(x, t)$ is plotted on a logarithmic scale in $(b))$ and $u(x, t)$, respectively, for varying $t=0$ to $t=10^{3}$, with $C a=10^{3}$, $K=1$ (the reference liquid viscosity $\mu_{0}^{\star}=K^{\star}$ ) and $R e=0$. At early times, the fluid in the film drains downwards (Fig. 2(c)) leading to thinning of the film in the upper region and a thickening in the lower region, and the film shape is concave-out (Fig. 2(a)). At late times, the fluid has drained significantly towards the lower end of the domain forming a quasi-static pendant drop there, leaving a very thin and almost flat film (lamella) in the middle region, and a quasi-static capillary meniscus at the upper end (Fig. 2(a)). This late-time behaviour can be clearly observed using a logarithmic scale for $h(x, t)$ shown in Fig. 2(b). This shows the middle lamella region connecting onto quasi-static curves at the top and bottom represented by the capillary meniscus and the pendant drop, respectively. The maximum flow speeds are in the middle lamella section of the film (Fig. 2(c)) which causes the film thickness to decrease severely there. The
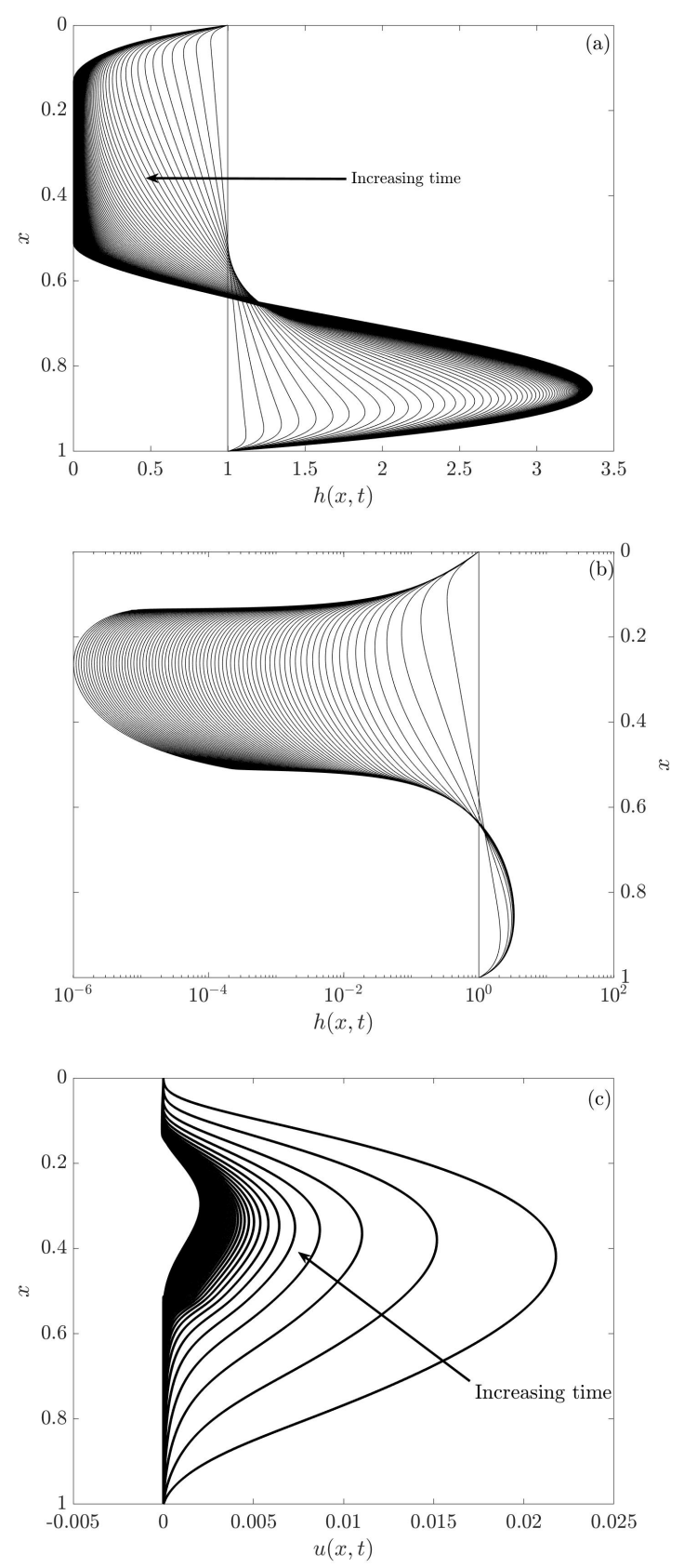

FIG. 2: The evolution of $(a),(b)$ the film thickness $h(x, t)((b)$ plots $h(x, t)$ on a logarithmic scale), and $(c)$ the extensional flow speed $u(x, t)$, for varying $t=0$ to $t=10^{3}$. The parameter values are: $n=1, C a=10^{3}$,

$$
K=1 \text { and } R e=0 \text {. }
$$

flow speed is zero near the top in the capillary meniscus region, and at the bottom in the pendant drop region.

It is instructive to describe the characteristic late-time flow and film evolution dynamics in transitions regions of very small width of $O(1 / C a$ ) near the top (where the upper meniscus meets the middle lamella) and bottom ends (where the middle lamella meets the pendant drop) of the film. The dynamics in these regions con- 
trol the liquid flux between the $O(1)$ lengthscale capillary menscii and the lamella, and hence influences the thinning and draining of the middle lamella region. Figure $3(a-c)$ show the evolution of the film thickness $h(x, t)$, the extensional flow speed $u(x, t)$ and the stress balance given by (14c) near the bottom end, respectively, for $t=(5,6,7,8,9,10) \times 10^{2}, n=1, C a=10^{3}$ and $R e=0$. We observe a similarity solution behaviour in the evolu-
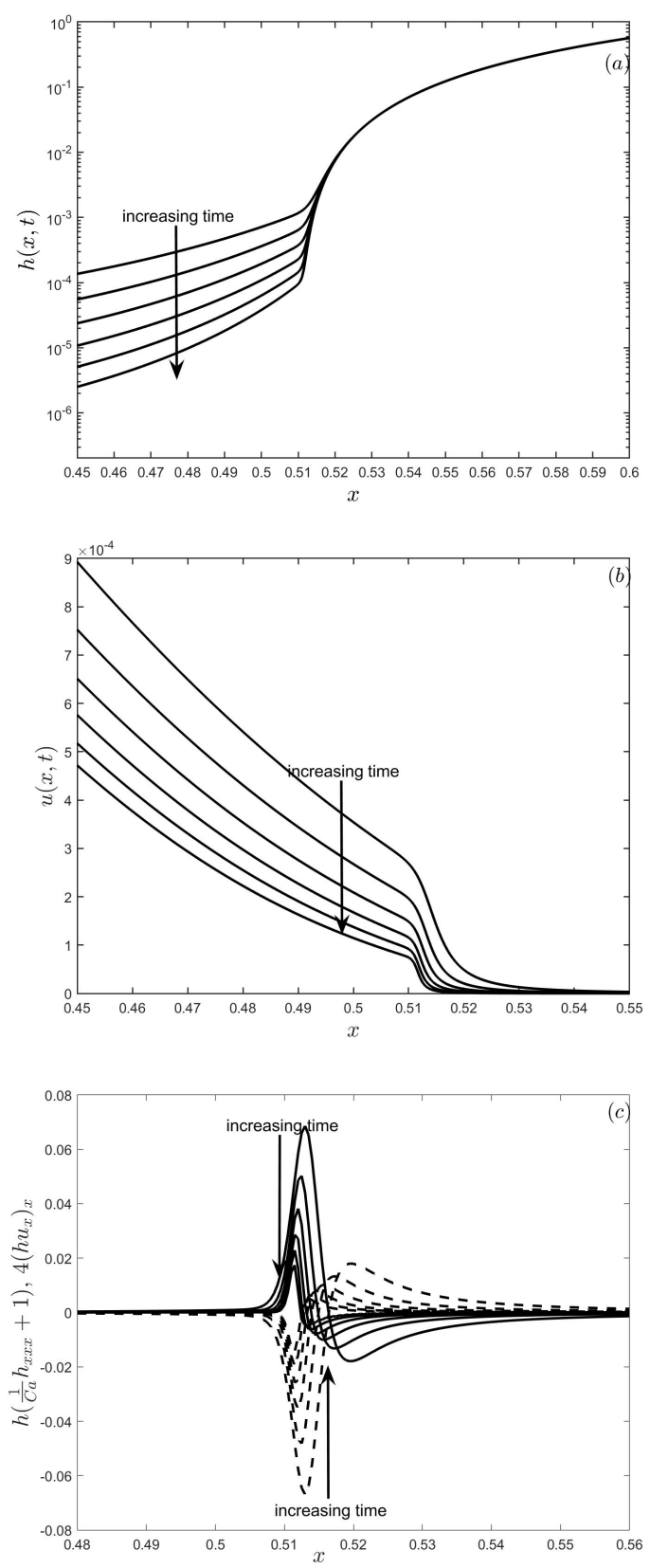

FIG. 3: The evolution of the $(a)$ film thickness $h(x, t)$, (b) extensional flow speed $u(x, t)$ and $(c)$ stress balance given by (14c) near the bottom end, for

$t=(5,6,7,8,9,10) \times 10^{2}$. The parameter values are: $n=1, C a=10^{3}, K=1$ and $R e=0$. tion of $h$ and $u$. The similarity solution behaviour of $h$ (see Fig. 3(a)) shows a family of solutions gradually thinning in time at its upstream end; at the downstream end, each solution connects onto a single quasi-static curve (represented by the pendant drop) at different locations (see Fig. 3(a)). The similarity solution behaviour of $u$ shows a characteristic jump (or shock-like behaviour) in $u$ at its downstream end (see Fig. 3(b)). This sudden deceleration of the flow is due to the sudden increase in film curvature (from almost zero in the lamella to $O(C a) \gg 1$ as the lamella connects onto the pendant drop). Indeed, the stress balance shown in Fig. 3(c) shows that the dominant contribution to the downward flow is due to the surface tension-related term, $h h_{x x x} / C a$ (shown by the solid black curves), which is dominant compared to the contribution to the downward flow due gravity in this region. This is balanced by the resistive (in this scenario) extensional stress term, $4\left(h u_{x}\right)_{x}$ (shown by the dashed curves). The resistive extensional stress results in the sudden deceleration of the flow. Fig. $4(a-c)$ show the evolution of the film thickness $h(x, t)$, the extensional flow speed $u(x, t)$ and the stress balance given by (14c) near the upper end, respectively, for $t=(5,6,7,8,9,10) \times 10^{2}$, $n=1, C a=10^{3}$ and $R e=0$. We also observe a similarity solution behaviour in the evolution of $h$ and $u$. The similarity solution behaviour of $h$ (see Fig. 4(a)) shows a family of solutions gradually thinning in time at its downstream end (much more severe thinning compared to that shown in Fig. 3(a)); at the upstream end, each solution connects onto a single quasi-static curve (represented by the capillary meniscus) at different locations. The similarity solution behaviour of $u$ shows a region where the flow is in the upward direction (see Fig. $4(b)$ ) where $u<0$ ). This reversal of the flow is due to the sudden increase in film curvature (from almost zero in the lamella to $C a \gg 1$ as the lamella connects onto the capillary meniscus). Indeed, the stress balance shown in Fig. 4(c) shows that the contribution due to the surface tension-related term, $h h_{x x x} / C a$ (shown by the solid black curves), dominates that due to gravity in this region. Moreover, $h h_{x x x} / \mathrm{Ca}$ is negative resulting in flow being sucked into the capillary meniscus leading to the reversal in flow. However, at the downstream end of this region the contribution of $h h_{x x x} / C a$ is negligible in comparison to gravity, resulting in downward flow (see solid black curves in Fig. 4(c)).

Figure $5(a, b)$ show the effect of increasing the capillary number, $C a=10^{2}, 10^{3}, 10^{4}$, for fixed $n=1$ and $R e=0$, on $h\left(x, t_{f}\right)$ and $u(x, t)$, respectively. Here $t_{f}$ is a fixed time for purposes of comparison, which is taken to be $10^{3}$ for $C a=10^{2}, 10^{3} ; t_{f}=339$ for $C a=10^{4}$, as the film thins rapidly for very large values of $C a$. We observe from Fig. $5(a)$ that as $C a$ increases the film thins more rapidly and the middle lamella section becomes much longer with a corresponding increase in the liquid collecting in the pendant drop at the bottom. As $\mathrm{Ca}$ increases, the effect of surface tension decreases in relative to gravity, thereby draining the film further. We 

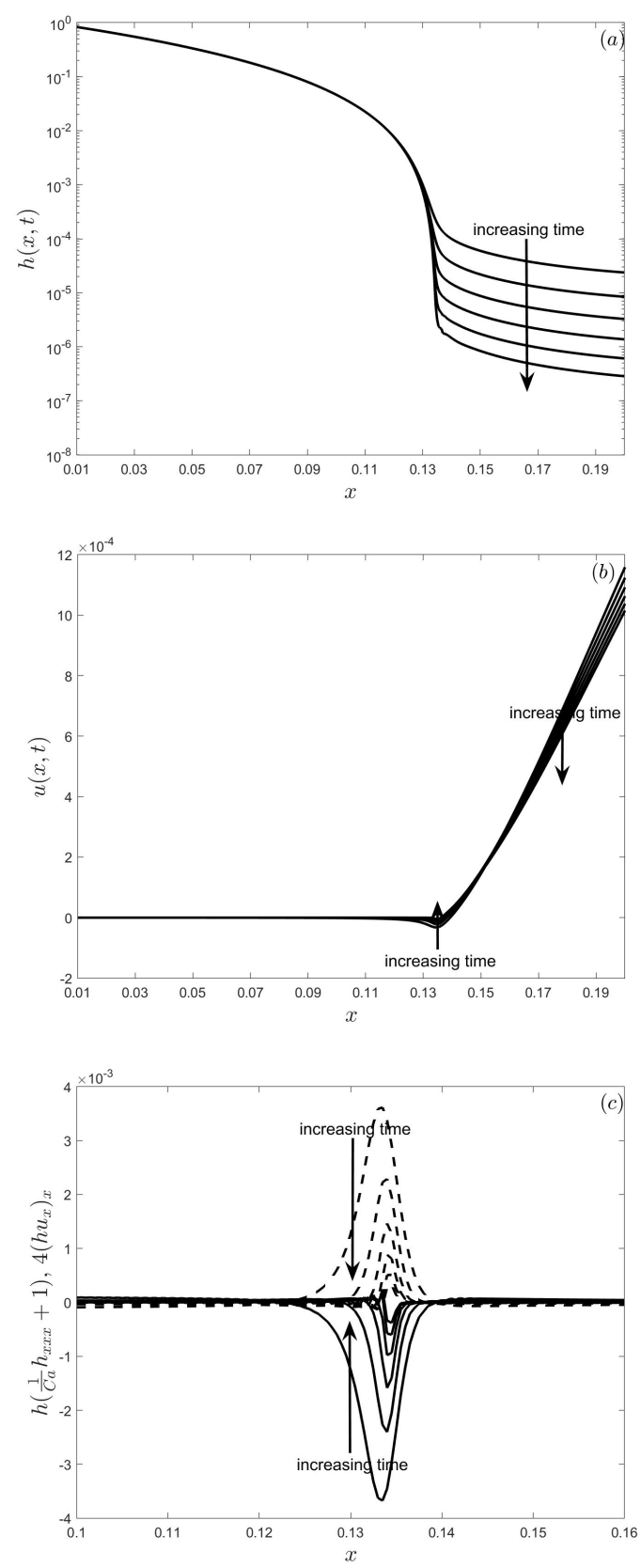

FIG. 4: The evolution of the $(a)$ film thickness $h(x, t)$, (b) extensional flow speed $u(x, t)$ and $(c)$ stress balance given by (14c) near the the upper end, for $t=(5,6,7,8,9,10) \times 10^{2}$. The parameter values are: $n=1, C a=10^{3}, K=1$ and $R e=0$.

also observe that the flow speed is much higher for larger values of $C a$ (Fig. 5(b)), resulting in faster drainage, and hence the film thins rapidly.

In Appendix B, we develop asymptotic solutions for the Newtonian case in the limit of $C a \gg 1$ describing the early and late-time quasi-steady and self-similar evolution behaviour displayed by the film and the extensional flow speed in the numerical simulations shown in Figs.
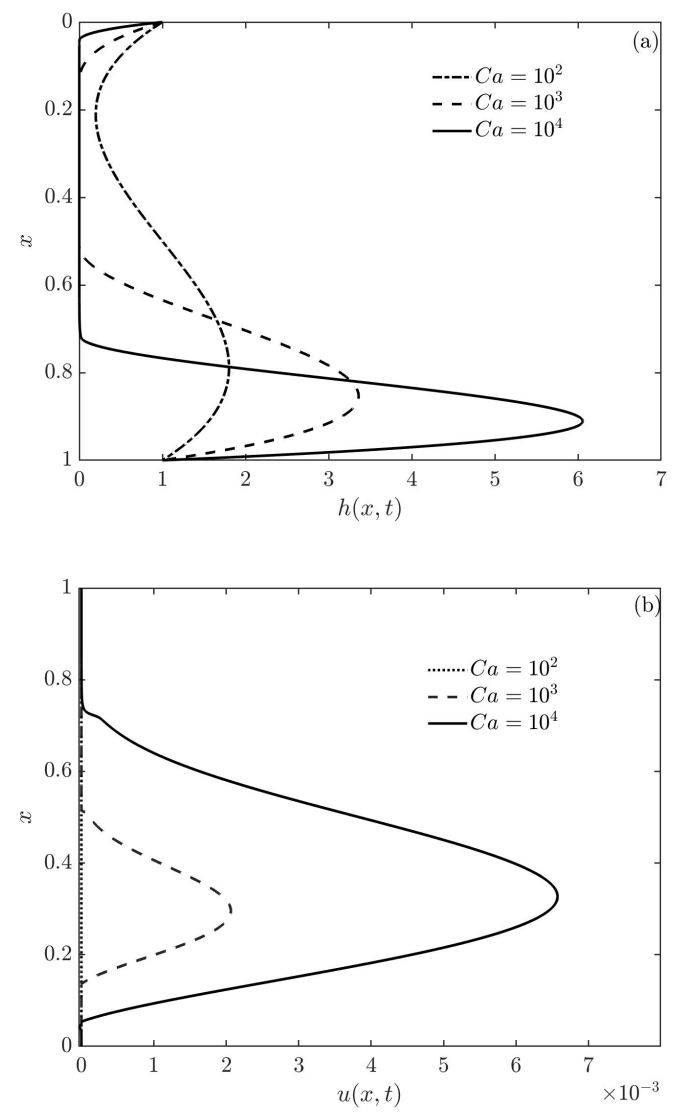

FIG. 5: (a) Film thickness $h\left(x, t=t_{f}\right)$, and $(b)$ extensional flow speed $u\left(x, t_{f}\right)$, for varying capillary number, $C a=10^{2}, 10^{3}, 10^{4}$, for $n=1, K=1, R e=0$ and $t_{f}=10^{3}$ for $C a=10^{2}, 10^{3} ; t_{f}=339$ for $C a=10^{4}$.

2-5. The early-time dynamics is described in Appendix B 1 based on the "growing exponential horn" similarity solution identified by Schwartz \& Roy ${ }^{16}$. The majority of the film exhibits a characteristic concave-out form (see Fig. 2( $a, b)$ at early times). This is due to the dominating extensional viscous flow, with the effect of surface tension confined to boundary layers near the ends (Fig. 2(a,b)). Figure $6(a, b)$ show a comparison between the numerical solution (Fig. 2(a,b)) at early times and the similarity solution for $h$ and $u$ (given by Eqs. B14, B12), respectively, for times $t=1,2,3,4,5,6,7$. Good agreement is observed at early time for $h$, except for the surface tension boundary layers near the top and bottom supports. As $t$ increases, the agreement is not so good. Good agreement is also observed at early time for $u$, except that the similarity solution slightly overestimates the corresponding numerical solution. This may be due to the influence of the boundary layers near the top and the bottom supports.

For late times, based on the observations from the numerical solutions shown in Figs. 2-5, we postulate a selfsimilar structure of the late-time evolution for $C a \gg 1$. The domain is divided into 5 regions (see Fig. 18 in 

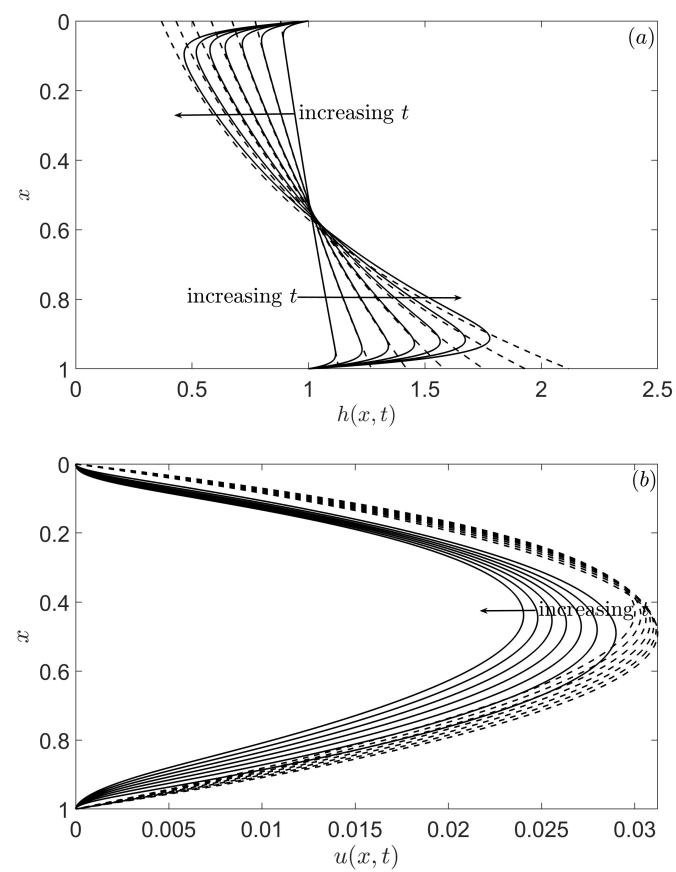

FIG. 6: Computed film thickness profiles, $h,(a)$ and extensional flow speeds, $u,(b)$ (solid lines) and the corresponding early-time similarity solution (dashed lines) given by Eqs. (B12,B14), for times,

$$
t=1,2,3,4,5,6,7 \text {. }
$$

Appendix B 2), comprising 3 long regions, Region I, the capillary meniscus, Region II, the middle lamella draining section and Region III, the pendant drop, and two short transition regions, A and B. In Appendix B 2 we describe the dominant physical mechanisms in each region and the corresponding equations, using which we derive semi-analytical approximate solutions. The quasi-steady evolution of each region is captured by the time evolution of characteristic variables, $x_{1,2}(t), h_{1,2}$ and $h_{m i n}(t)$ (see Appendix B 2 for their definitions). Finally, we derive closure relationships based on global volume conservation which allows the time evolution of the characteristic variables to be determined.

The late-time self-similar solution structure reveals the relationship between $Q_{1,2}$, the fluxes from the lamella (Region II) to the upper plateau border (Region I) and the lower plateau border region (upstream part of Region III), respectively, and $x_{2}-x_{1}$, the width of the lamellar region (see Appendix B 2 for details). The fluxes $Q_{1,2}$ are shown to contribute to increasing the volume of the plateau border regions; the increased volume reduces the width $x_{2}-x_{1}$, which in turn influences $Q_{1,2}$ via the dynamics in the transition regions (Regions $\mathrm{A}$ and B). An approximate analysis of the lamella shows that its minimum thickness $h_{\text {min }} \sim Q_{2} /\left(x_{2}-x_{1}\right)^{2}$, while the corresponding maximum in extensional flow speed $u_{\max } \sim\left(x_{2}-x_{1}\right)^{2}$. We develop an algorithm to determine the relationship between $Q_{2}$ and $x_{2}-x_{1}$ nu- merically, however, we are unable to derive an analytical relationship to determine a power-law thinning rate analytically using the above relationships for $\left(h_{\min }, u_{\max }\right)$. It is worth noting here that the theoretical analysis by Breward $^{23,24}$ and Brush \& Davis ${ }^{25}$ is based on determining the functional relationship $Q_{2}\left(h_{\min }\right)$. In their analysis, without including gravity, Eq. (B56) in Region B can be integrated, and the relationship $Q_{2}\left(h_{m i n}\right)$ is obtained using the boundary conditions. Moreover, without the inclusion of gravity, the extensional flow in the lamellar region (Region I) is linear, hence the film thickness $h$ in this region is a time-dependent constant. One can then solve the differential equation in Eq. (B22) to obtain a thinning rate, $h_{\text {min }, t} \sim t^{-2}$ determined by Breward ${ }^{23,24}$. However, quasi-steady nature of the problem and the inclusion of gravity precludes the derivation of such analytical solutions. Nevertheless, using the numerical simulations shown in Fig. 1, we can compute $h_{m i n}(t)$ - the global minimum in $h$ - which is attained in the middle lamella section of the film. The red curve in Fig. 9(c) shows the time evolution of $h_{\min }(t)$ on a logarithmic scale for a Newtonian liquid $(n=1)$. Using this a power-law thinning rate of $t^{-2.25}$ is predicted, in comparison to the $t^{-2}$ power-law behaviour in the absence of gravity. Although this is a weak dependence of the thinning rate on gravity, it suggests the long-lived influence of gravity still persists even after it becomes very thin. This prediction holds until the dimensionless film thickness is $O\left(10^{-3}\right)$ below which the film thins rapidly and the numerical solution breaks down. Additional physics, such as van der Waals forces, need to be included to capture the appropriate behaviour at these small thicknesses.

In the next set of results, we show the influence of varying the liquid consistency index $K$ (or dimensional $K^{\star}$ ). This can also be analysed by choosing $K=1$, so that the reference liquid viscosity, $\mu_{0}^{\star}=K^{\star}\left(2 U^{\star} / L^{\star}\right)^{n-1}$ or $\mu_{0}^{\star}=K^{\star^{1 / n}}\left(2 \rho^{\star} g^{\star} L^{\star}\right)^{(n-1) / n}$, for a liquid of given consistency $K^{\star}$. The characteristic speed and time of draining of the flow and thinning of the film can then be shown to scale with $K^{\star}$ as $U^{\star} \sim 1 / K^{\star^{1 / n}}$ and $t^{\star} \sim K^{\star^{1 / n}}$, respectively. We choose not to scale $K$ out of the results shown here by using the above scalings in order to explicitly investigate its influence. This will be useful in the results to follow. Fig. $7(a, b, c)$ show the effect of varying $K$ on $h(x, t=30)$ and $u(x, t=30)$ and $h_{m i n}(t)$, respectively, for $n=1, C a=10^{3}$ and $R e=0$. The time $t=30$ is chosen arbitrarily so as to capture the evolution for low values of $K$ before the film thins below a threshold value and the numerical solution breaks down. We observe that the film thins more rapidly as $K$ decreases (Fig. $7(a)$ ) due to the faster extensional flow speed as $K$ decreases (Fig. $7(b))$. Reducing $K$ leads to lower viscosities, resulting in much faster drainage (note that $\left.u \sim 1 / K^{1 / n}\right)$. Figure $7(c)$ tracks $h_{\text {min }}$, the global minimum in $h(x, t)$, as a function of time $t$. We observe that the film thins more rapidly as $K$ decreases, in line with the observations in Fig. $7(a, b)$. We also note that the time scale for thinning scales like $t \sim K^{1 / n}$. To bet- 

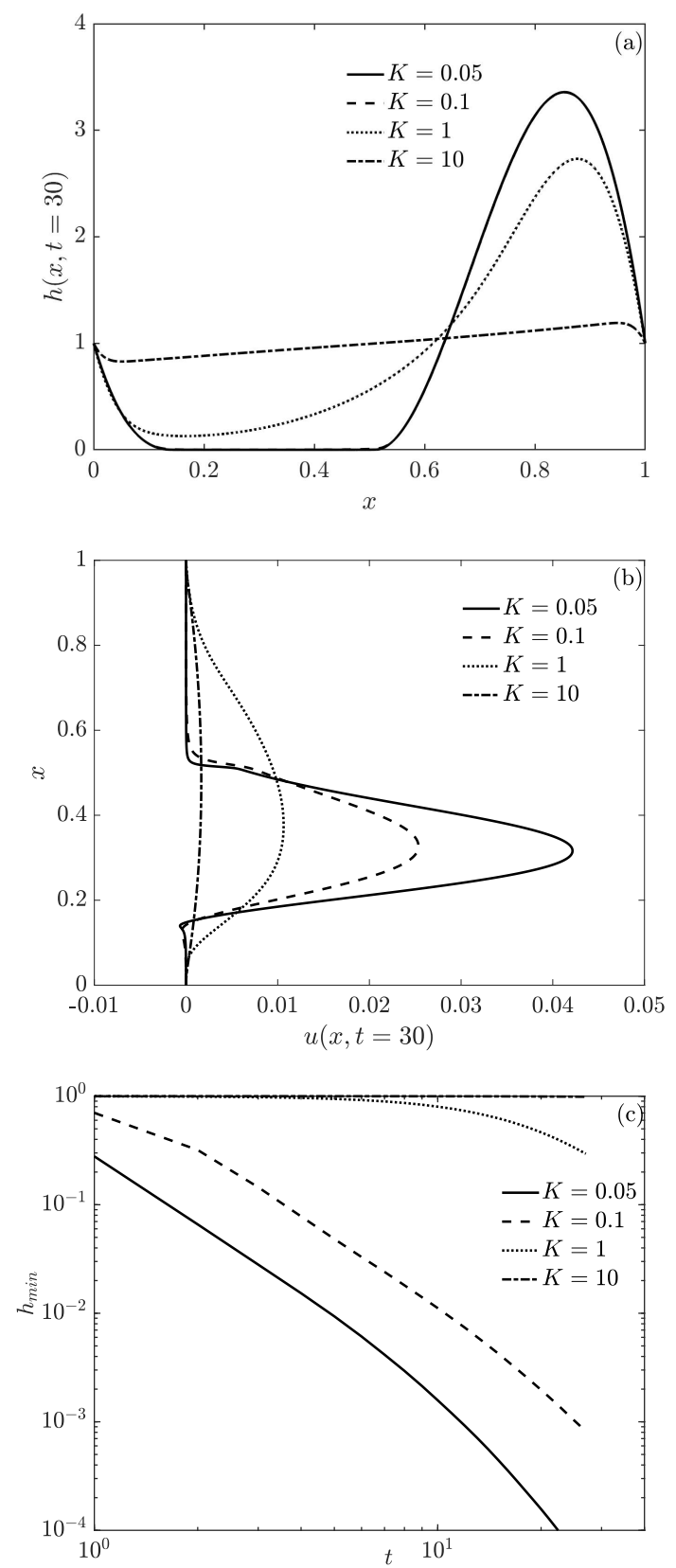

FIG. 7: Film thickness $h(x, t=30)$, and $(b)$ extensional flow speed $u(x, t=30)$, and (c) the global minimum $h_{\min }$ as a function of time $t$, for varying consistency parameter $K$. The parameters are: $n=1, C a=10^{3}$ and $R e=0$.

ter understand the above described variations in $K$, we plot key outputs, $h(x, t=30), u(x, t=30)$, the shearrate $u_{x}(x, t=30)$ and the shear-rate dependent viscosity $\mu\left(\left|u_{x}\right|\right)$ for $K=0.02$ (corresponding to a liquid of a very low consistency index) in Fig. 8( $a), K=0.1$ (corresponding to a liquid of a low consistency index) in Fig. 8(b), $K=1$ (corresponding to a liquid of an intermediate consistency index) in Fig. 8(c) and $K=10$ (corresponding to a liquid of a high consistency index) in Fig. $8(d)$, for fixed $n=1, C a=10^{3}$ and $R e=0$. We observe that the magnitude of shear rate $\left|u_{x}\right|$ is larger for the lower values of $K$ (Fig. 8(a,b)), in comparison to the higher values of $K$ (Fig. $8(c, d)$ ). We also observe a sharp peak in $u_{x}$ for $K=0.02$ concentrated in the transition region where the lamella connects onto the pendant drop region near the bottom (Fig. 8(a)), which progressively becomes smaller as $K$ increases (Fig. $8(b-e)$ ). This results in lower viscosities for smaller $K$ (Fig. 8( $(a, b))$ and higher viscosities as $K$ increases (Fig. $8(c, d)$ ).

Next, we consider the case of a non-Newtonian liquid. We first show the influence of varying the powerlaw index $n$. We choose $\mu_{0}^{\star}=K^{\star}$ (by choosing $n=$ 1 or a Newtonian liquid reference viscosity). Then $K(n)=\left(U^{\star} / L^{\star}\right)^{n-1}=\left(\rho^{\star} g^{\star} L^{\star} / K^{\star}\right)^{n-1}=K_{0}^{n-1}$. Fig. $9(a, b)$ show the effect of varying the power-law index, $n=0.75,1,1.2$, on $h(x, t=360)$ and $u(x, t=360)$, respectively, for fixed $K_{0}=1, C a=10^{3}$ and $R e=0$. We observe that the film thins more rapidly as $n$ increases (Fig. 9(a)) due to the faster extensional flow speed as $n$ increases (Fig. 9(b)). Note Fig. 9(a) only shows the middle lamella region as the upper and lower parts of the film are not significantly affected by varying $n$. Figure $9(c)$ tracks $h_{\min }$, the global minimum in $h(x, t)$, as a function of time $t$, for $n=0.6,0.65,0.75,0.85,1,1.1,1.2$. We observe that the film thins more rapidly as $n$ increases, in line with the observations in Fig. $9(a, b)$. We estimate the thinning rates to be $t^{-2}$ when $n<1, t^{-2.25}$ when $n \geq 1$, suggesting a weak dependence of the thinning rate on $n$. This power-law estimate is at best valid near $n=1$ as shown in Fig. 9(c), where the range of powerlaw behaviour reduces as $n$ moves further away from one. To better understand the above described variations in $n$, we plot key outputs, $h(x, t=360), u(x, t=360)$, the shear-rate $u_{x}(x, t=360)$ and the shear-rate dependent viscosity $\mu\left(\left|u_{x}\right|\right)$ for $n=0.75$ (corresponding to the shear-thinning fluid case) in Fig. $10(a), n=1$ (corresponding to the Newtonian fluid case) in Fig. 10(b) and $n=1.25$ (corresponding to the shear thickening fluid case) in Fig. $10(c)$, for fixed $K_{0}=1, C a=10^{3}$ and $R e=0$. We observe that the shear rate $u_{x} \ll 1$ across the entire film, and throughout the evolution process, for the value of $K_{0}\left(K^{\star}\right)$ investigated here. This results in higher viscosities for $n<1$ (Fig. 10(a)) and lower viscosities for $n \geq 1$ (Fig. 10(b,c)). Moreover, we observe from Fig. 10(a) that the spatial profile of viscosity for $n<1$ is maximum near the top, bottom and lamella regions where the shear rate $u_{x}=0$. The opposite happens for $n>1$ (Fig. 10(c)), with a spike in viscosity corresponding to a change in shear rate observed where the flat middle region of the film connects onto the pendant drop near the bottom of the film (see Fig. 10(b)). We note here that Power-law model is not accurate at very low shear rates (see Myers ${ }^{29}$ ) as observed here, hence we need to be careful in interpreting the above results. In order to further investigate the influence of varying $n$, we consider a much higher value of $K_{0}=30$ (which 

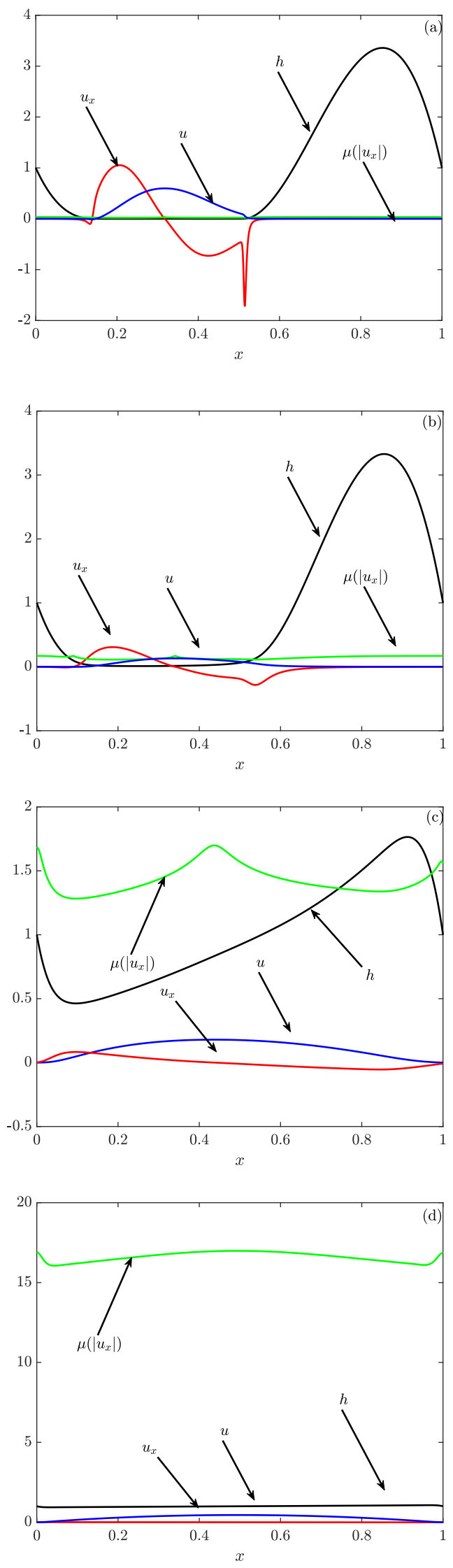

FIG. 8: The film thickness $h(x, t=30)$ (black curve), the extensional flow speed $u(x, t=30)$ (blue curve), the shear-rate $u_{x}(x, t=30)$ (red curve) and the shear-rate dependent viscosity $\mu\left(\left|u_{x}\right|\right)$ (green curve) for $(a)$ $K=0.02$, (b) $K=0.1$, (c) $K=1$ and $(d) K=10$. The parameter values are: $n=1, C a=10^{3}$ and $R e=0$.
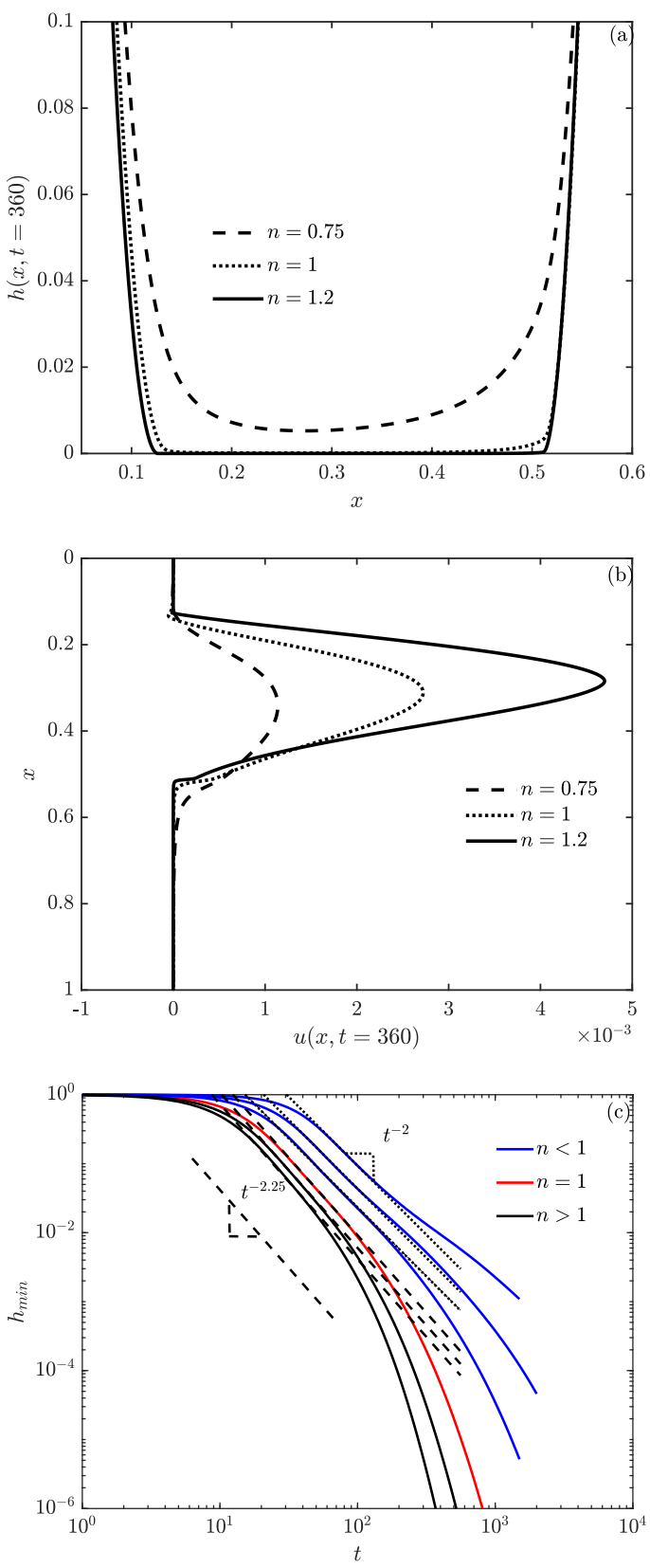

FIG. 9: Film thickness $h(x, t=360)$ and $(b)$ extensional flow speed $u(x, t=360)$ for varying power-law index $n$, and $(c)$ the global minimum $h_{\min }$ as a function of time $t$ for varying power-law index, $n=0.6,0.65,0.75,0.85,1,1.1,1.2$. The parameters are: $K_{0}=1, C a=10^{3}$ and $R e=0$. The corresponding thinning rates are $t^{-2}$ for $n<1$ and $t^{-2.25}$ for $n \geq 1$.

corresponds to smaller consistency index $K^{\star}$ ). The resulting flow is of similar mobility and the shear rates are also similar to the previous case with $K_{0}=1$. Moreover, $K(n)=K_{0}^{n-1}$, depends on $n$, which was not the case before for $K_{0}=1$. So, the influence on $n$ will now come from $\mu\left(\left|u_{x}\right|\right)=K_{0}^{n-1}\left|u_{x}\right|^{n-1}$. So, if the shear rates are 

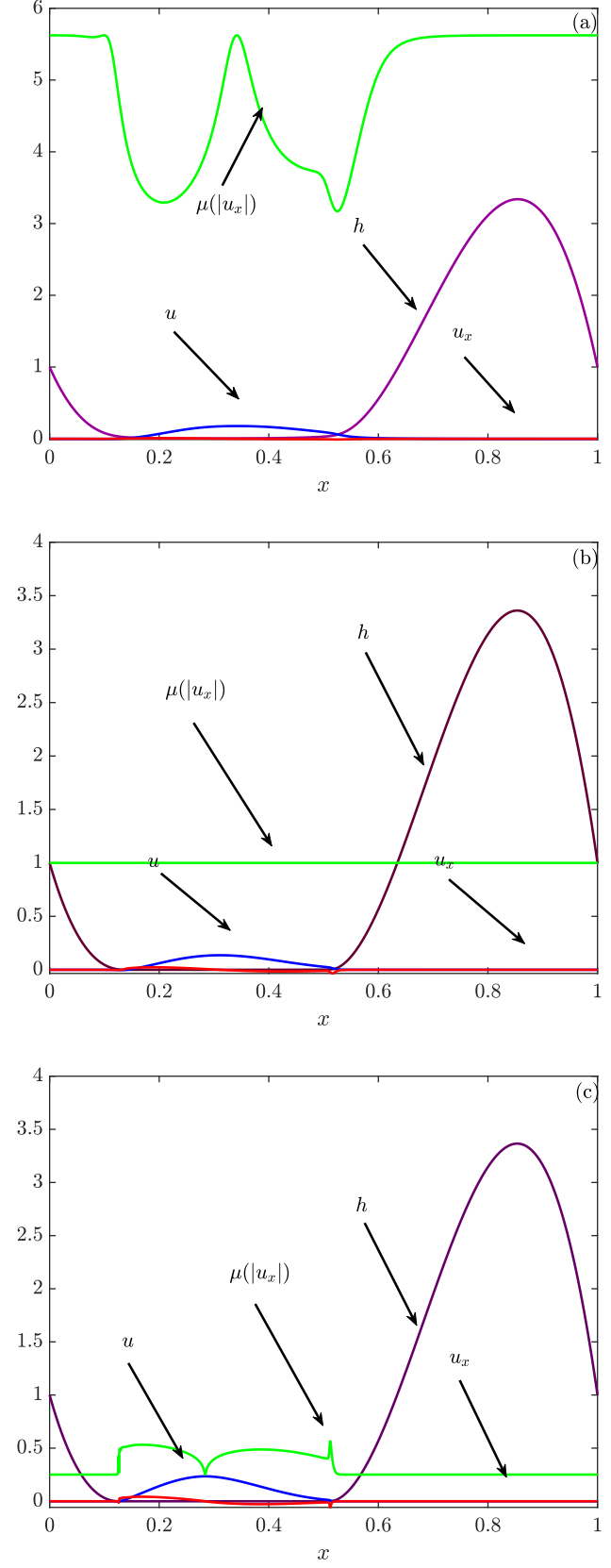

FIG. 10: The film thickness $h(x, t=360)$ (black curve), the extensional flow speed $u(x, t=360)$ (blue curve),

the shear-rate $u_{x}(x, t=360)$ (red curve) and the shear-rate dependent viscosity $\mu\left(\left|u_{x}\right|\right)$ (green curve) for (a) $n=0.75$ (corresponding to the shear-thinning fluid case), (b) $n=1$ (corresponding to the Newtonian fluid case), and $n=1.25$ (corresponding to the shear-thickening fluid case). The parameters are: $K_{0}=1, C a=10^{3}$ and $R e=0$. low and $n<1$, then the higher value of $\left|u_{x}\right|^{n-1}$ could be offset by the lower value of $K_{0}^{n-1}$ (which is approximately 0.5 for $n=0.9$ ), resulting in a lower viscosity $\mu\left(\left|u_{x}\right|\right)$. In contrast for $n>1$, then $\left|u_{x}\right|^{n-1}$ is small, but $K_{0}^{n-1}$ is large (which is approximately 2 for $n=1.2$ ), which could result in a higher viscosity $\mu\left(\left|u_{x}\right|\right)$. An illustration of this behaviour is observed in Fig. 11, which plots $h_{\min }$ versus $t$ for $K_{0}=30$, and $n=0.85,1,1.2$. We observe that at early to intermediate times during

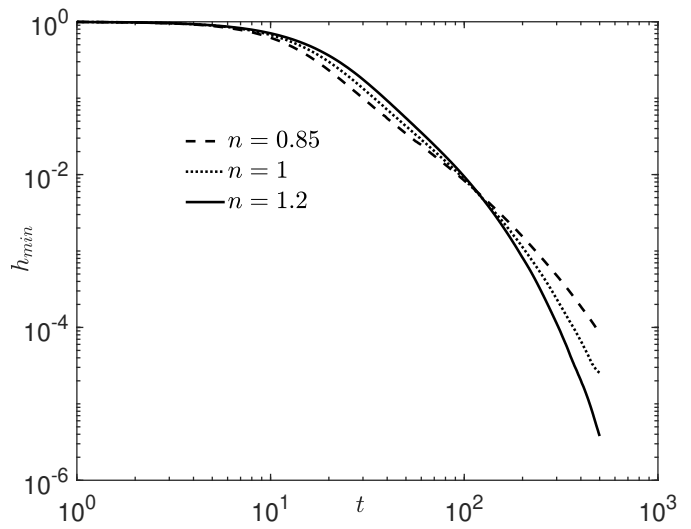

FIG. 11: The global minimum $h_{\min }$ as a function of time $t$, for varying power-law index, $n=0.85,1,1.2$, for fixed $K_{0}=30, C a=10^{3}$ and $R e=0$.

the draining process, $h_{\min }$ thins faster for $n=0.85 \mathrm{com}$ pared to $n=1.2$. This is due to the faster drainage for $n<1$ at the given value of $K_{0}=30$, in comparison to a relatively slower drainage for $n>1$ (results not shown). At later times, the roles are reversed and the thinning behaviour is similar to the case corresponding to $K_{0}=1$. This is due to the fact that the shear rates have become very small, so that the $K_{0}^{n-1}$ can no longer offset the $\left|u_{x}\right|^{n-1}$ term, which is very large for $n<1$ and very small for $n>1$. If we were to further increase $K_{0}$, then the thinning behaviour observed at early and intermediate times would persist for much longer times.

The Power-law model might not accurately reflect the dynamics of the low shear rate cases ${ }^{29}$. We now investigate the Carreau model which is well behaved at low shear rates. Moreover, no regularisation is required at low shear rates for $n<1$ either, unlike in the Powerlaw model. In the simulations to follow, we investigate the Carreau model for varying $n$, with $\mu_{1}=1$ (so the reference viscosity is chosen to be the zero shear rate viscosity), $C a=10^{3}, \lambda=100\left(\lambda^{\star}=10 \mathrm{~s}\right)$ and $\mu_{\infty}=0$ fixed. (Note that $\mu_{\infty} \ll 1$.) Figure $12(a, b)$ show the effect of varying the power-law index, $n=0.75,1,1.2$, for $C a=$ $10^{3}$ and $R e=0$, on $h(x, t=800), u(x, t=800)$. We observe that the film thins more rapidly as $n$ decreases (Fig. 12(a)) due to the faster extensional flow speed as $n$ decreases (Fig. 12(b)). The low flow shear rates during the evolution result in lower viscosities for $n<1$ (shear thinning) and higher viscosities for $n>1$ (shear thicken- 

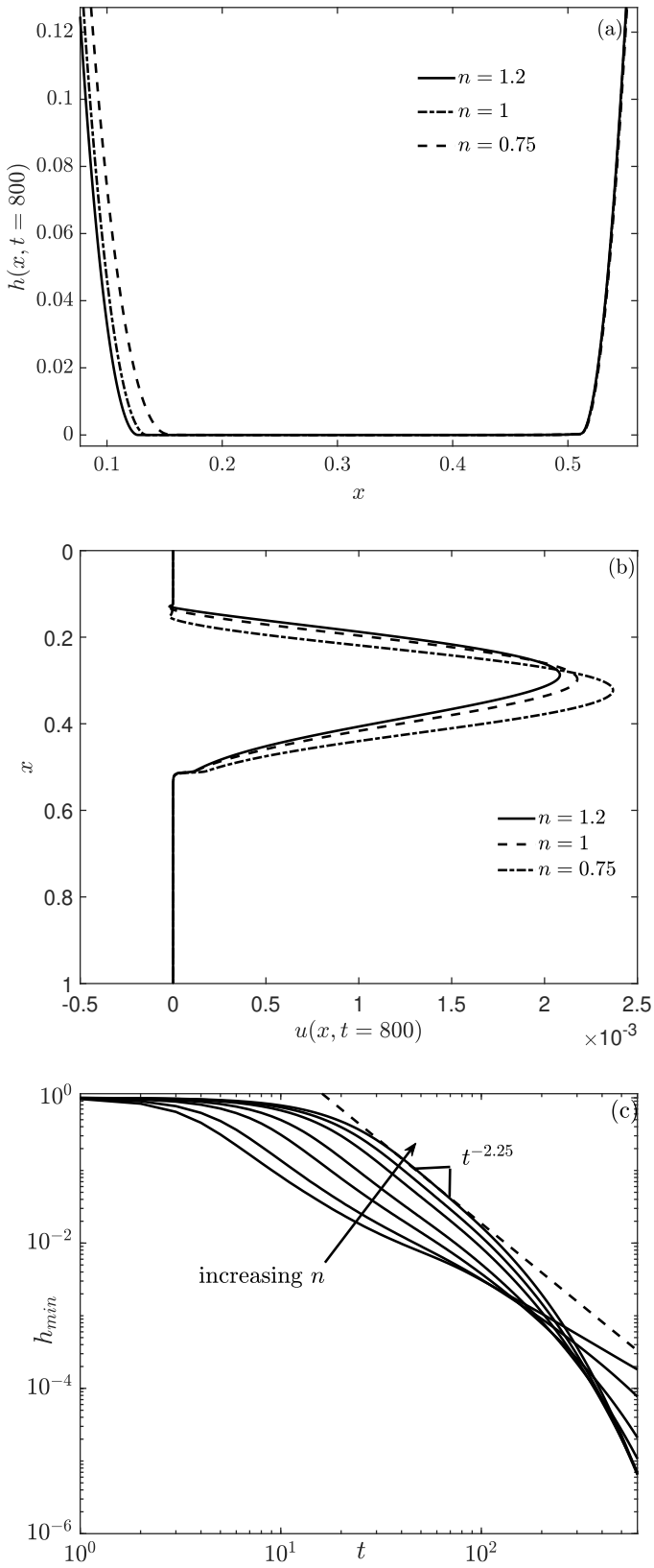

FIG. 12: (a) Film thickness $h(x, t=800),(b)$ extensional flow speed $u(x, t=800)$ and $(c)$ the global minimum $h_{\text {min }}$ as a function of time $t$, for the Carreau model with $n=0.6,0.65,0.75,0.85,1,1.1,1.2, \mu_{1}=1$, $C a=10^{3}, \lambda=100\left(\lambda^{\star}=10 \mathrm{~s}\right), \mu_{\infty}=0$ and $R e=0$ fixed. We estimate the thinning rate to be $t^{-2.25}$ for $n<1$ and $n \geq 1$.

ing), resulting in much slower drainage for $n>1$. Figure $12(c)$ tracks $h_{\text {min }}$, the global minimum in $h(x, t)$, as a function of time $t$, for $n=0.6,0.65,0.75,0.85,1,1.1,1.2$. We observe that the film thins more rapidly as $n$ decreases, in line with the observations in Fig. 12(a,b). We estimate the thinning rate to be $t^{-2.25}$ for $n \geq 1$ and $n<1$. To better understand the variations in $n$, we plot key outputs, $h(x, t=800), u(x, t=800)$, the shear-rate $u_{x}(x, t=800)$ and the shear-rate dependent viscosity $\mu\left(\left|u_{x}\right|\right)$ for $n=0.75$ (corresponding to the shear-thinning fluid case) in Fig. 13(a), and $n=1.2$ (corresponding to the shear thickening fluid case) in Fig. 13(b). We ob-
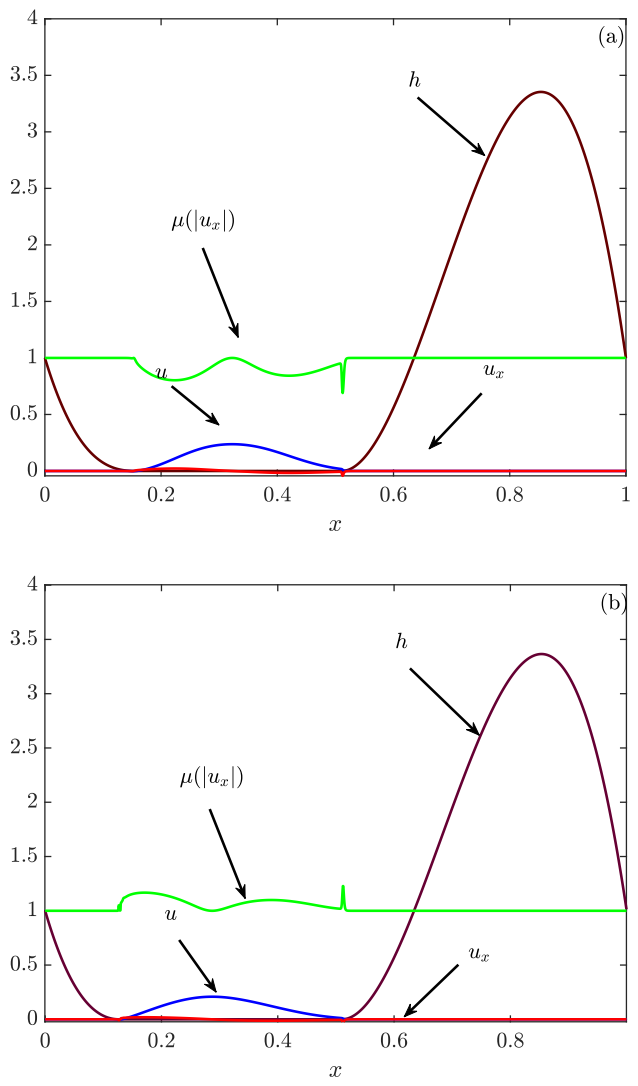

FIG. 13: The film thickness $h(x, t=800)$ (black curve), the extensional flow speed $u(x, t=800)$ (blue curve),

the shear-rate $u_{x}(x, t=800)$ (red curve) and the shear-rate dependent viscosity $\mu\left(\left|u_{x}\right|\right)$ (green curve) for (a) $n=0.75$ (corresponding to the shear-thinning fluid case), and (b) $n=1.2$ (corresponding to the shear-thickening fluid case), for fixed $\mu_{1}=1, C a=10^{3}$, $\lambda=100\left(\lambda^{\star}=10 \mathrm{~s}\right), \mu_{\infty}=0$ and $R e=0$.

serve that the shear rate $u_{x} \ll 1$ across the entire film, and throughout the evolution process. This results in lower viscosities for $n<1$ (Fig. 13(a)) and higher viscosities for $n \geq 1$ (Fig. 13(b)). Moreover, we observe from Fig. 13(a) that the spatial profile of viscosity for $n<1$ is less than 1 near where the shear rate $u_{x} \neq 0$. The opposite happens for $n>1$ (Fig. 13(b)), with a spike in viscosity corresponding to a change in shear rate observed where the middle lamella region connects onto the pendant drop near the bottom of the film.

We now consider the effects of varying the yield stress $\tau_{p}$ on the evolution using the regularised HerschelBulkley constitutive model. The values of $\tau_{p}$ chosen are 
informed based on the absolute value of the extensional stress profile $\left|\tau_{x x}^{x}\right|=\left|u_{x}^{n}\right|$ determined from the initial shear rate $u_{x}(x, t=0)$ corresponding to the initial profile of $u$ given by Eq. (17). The maximum value of the initial stress $\tau_{x x}^{x}$ then provides the maximum value of $\tau_{p}$; the flow will not yield from its initial state for any value of $\tau_{p}$ greater than this maximum value; any value of $\tau_{p}$ less than this maximum value will result in sections of the flow yielding while others do not yield (weakly yielding for the regularised form of the Herschel-Bulkley constitutive model). We explore this below for varying power-law index $n$. Figure $14(a, b, c)$ show the effect of varying the yield stress $\tau_{p}=10^{-4}, 5 \times 10^{-3}, 10^{-2}, 2 \times 10^{-2}, 4 \times 10^{-2}$, for $n=0.75, K_{0}=1, C a=10^{3}$ and $R e=0$, on $h\left(x, t=10^{3}\right), u\left(x, t=10^{3}\right)$, and the global minimum $h_{\text {min }}$ as a function of time $t$, respectively. We observe in Fig. 14(b) that increasing the yield stress $\tau_{p}$ slows $u$ and exhibits weakly yielding behaviour for large $\tau_{p}$. This results in the central region of the film to be much thicker near the top and the bottom as $\tau_{p}$ is increased, as observed in Fig. 14(a). Indeed, Fig. 14(c) shows that the film's minimum thickness $h_{m i n}$ increases slightly as $\tau_{p}$ increases, for any given time $t$. We estimate the thinning rates to be $t^{-2}$ for $\tau_{p}=10^{-4}$ and $t^{-1.8}$ for $\tau_{p}=2 \times 10^{-2}$. This suggests that the thinning rate decreases as $\tau_{p}$ increases. A weak dependence of the yield stress on the thinning rate is observed when measured against the zero yield stress thinning rate of $t^{-2}$ for $n=0.75$. Fig. $15(a, b, c)$ show the effect of varying the yield stress $\tau_{p}=2 \times 10^{-2}, 10^{-2}, 5 \times 10^{-3}, 10^{-4}$, for $n=1, K_{0}=1$, $C a=10^{3}$ and $R e=0$, on $h\left(x, t=10^{3}\right), u\left(x, t=10^{3}\right)$, and the global minimum $h_{\min }$ as a function of time $t$, respectively. Fig. $15(a, b, c)$ show similar behaviour and trends as in the previous case for $n=0.75$, except that $u$ (and the corresponding shear rate $u_{x}$ and stress $\tau$ ) are larger as $n$ increases (note that the maximum $u$ is much higher than that for $n=0.75$, and at later time). We estimate the thinning rates to be $t^{-2.25}$ for $\tau_{p}=10^{-4}$ and $t^{-1.7}$ for $\tau_{p}=2 \times 10^{-2}$. This suggests that the thinning rate decreases as $\tau_{p}$ increases. A weak dependence of the yield stress on the thinning rate is observed when measured against the zero yield stress thinning rate of $t^{-2.25}$ for $n=1$. Fig. $16(a, b, c)$ show the effect of varying the yield stress $\tau_{p}=1.6 \times 10^{-2}, 10^{-2}, 5 \times 10^{-3}, 10^{-4}$, for $n=1.2, K_{0}=1, C a=10^{3}$ and $R e=0$, on $h(x, t=360)$, $u(x, t=360)$, and the global minimum $h_{\min }$ as a function of time $t$, respectively. Fig. 16( $a, b, c)$ again show similar behaviour and trends as in the previous two cases for $n=0.75,1$, except that $u$ (and the corresponding shear rate $u_{x}$ and stress $\tau$ ) are much larger as $n$ increases. In figure $16(c)$, we estimate the thinning rates to be $t^{-2.35}$ for $\tau_{p}=10^{-4}$ and $t^{-2}$ for $\tau_{p}=1.6 \times 10^{-2}$. This suggests that the thinning rate decreases as $\tau_{p}$ increases. A weak dependence of the yield stress on the thinning rate is observed when measured against the zero yield stress thinning rate of $t^{-2.35}$ for $n=1.2$.
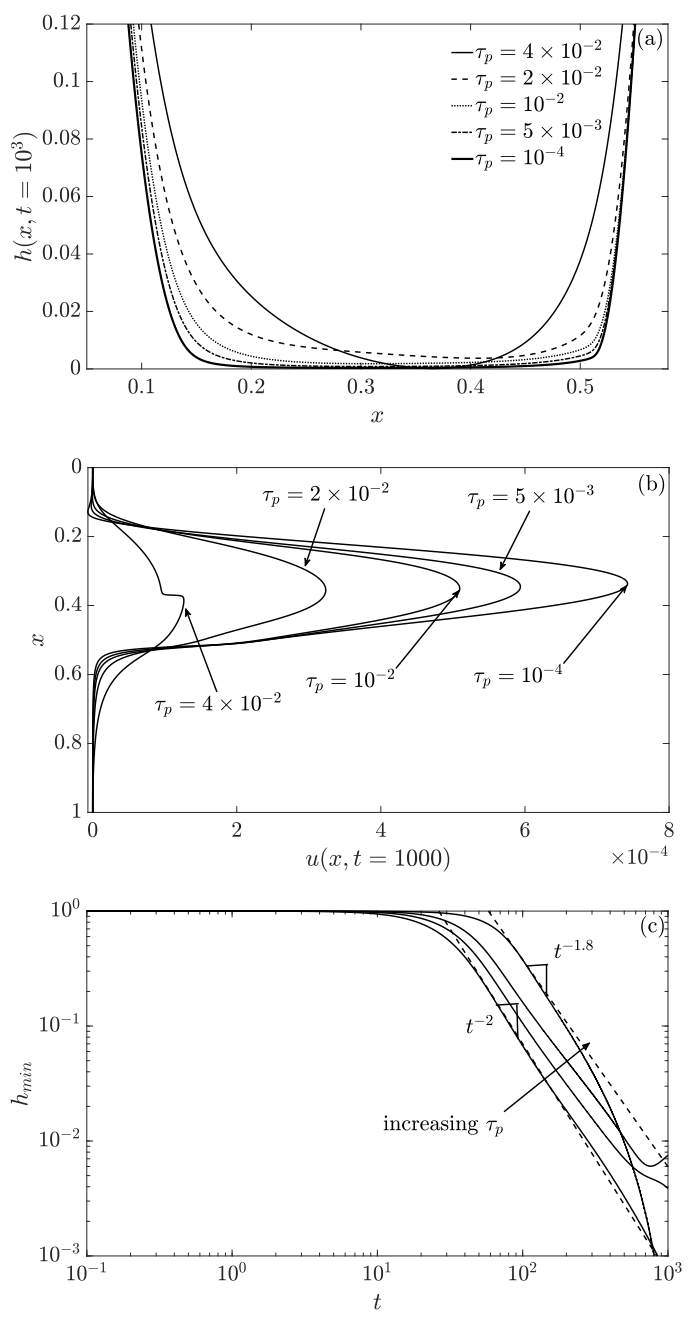

FIG. 14: (a) Film thickness $h\left(x, t=10^{3}\right)$, and $(b)$ extensional flow speed $u\left(x, t=10^{3}\right)$, and $(c)$ the global minimum $h_{\min }$ as a function of time $t$, for varying yield stress $\tau_{p}=10^{-4}, 5 \times 10^{-3}, 10^{-2}, 2 \times 10^{-2}, 4 \times 10^{-2}$, for $n=0.75, K_{0}=1, C a=10^{3}$ and $R e=0$. The corresponding thinning rates are $t^{-2}$ for $\tau_{p}=10^{-4}$ and $t^{-1.8}$ for $\tau_{p}=2 \times 10^{-2}$.

\section{CONCLUSION}

In this paper, we investigated the draining of a vertically-aligned free non-Newtonian liquid film between two rigid supports due to the combined effects of extensional viscous, gravity and surface tension forces. Our numerical simulations and asymptotic analysis have focussed on the case when the rescaled capillary number $C a=\hat{C a} a / \epsilon \gg 1$, where the capillary number $\hat{C a}=$ $\mu^{\star} U^{\star} / \gamma^{\star}=O(1)$. This limit recreated the decomposition of the liquid domain into a lamella connecting onto plateau borders, which replicates the drainage due to gravity in liquid foams ${ }^{23-25}$.

We considered the draining of initially thick films and focussed on the dominant balance between the exten- 

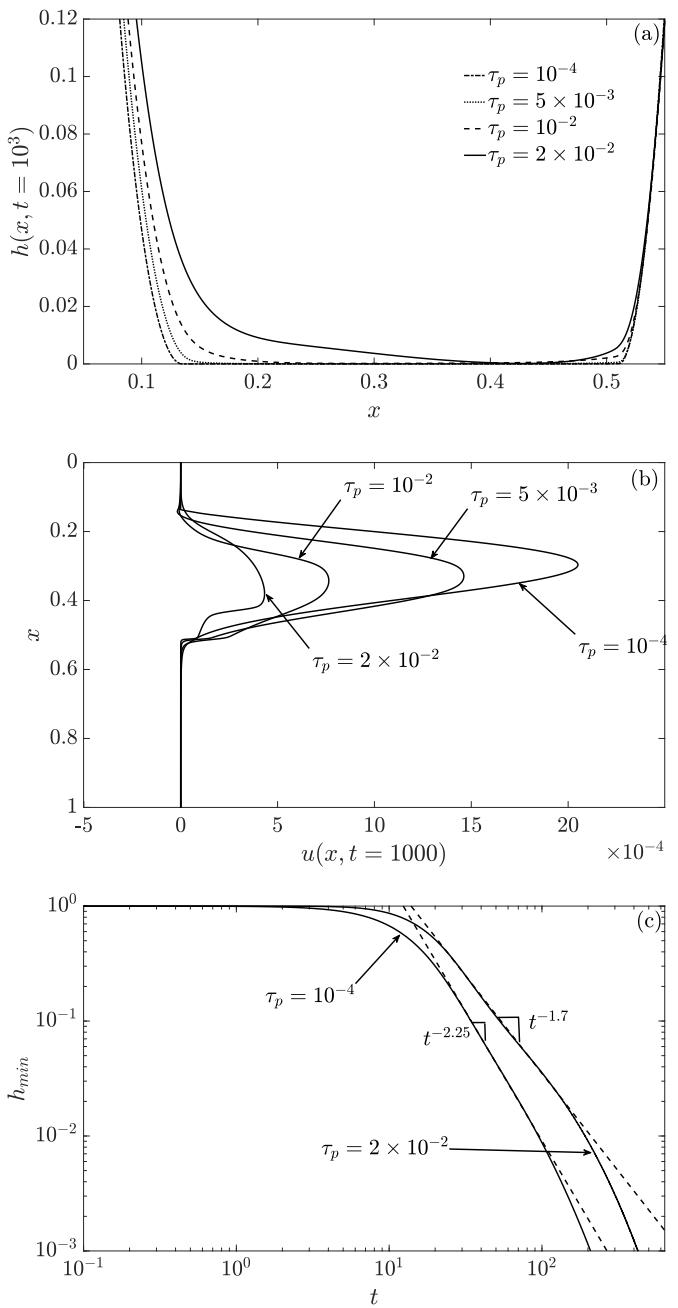

FIG. 15: (a) Film thickness $h\left(x, t=10^{3}\right)$, and $(b)$ extensional flow speed $u(x, t=)$, and $(c)$ the global minimum $h_{\min }$ as a function of time $t$, for varying yield stress $\tau_{p}=2 \times 10^{-2}, 10^{-2}, 5 \times 10^{-3}, 10^{-4}$, for $n=1$, $K_{0}=1$ and $C a=10^{3}$. The corresponding thinning

rates are $t^{-2.25}$ for $\tau_{p}=10^{-4}$ and $t^{-1.7}$ for

$$
\tau_{p}=2 \times 10^{-2} .
$$

sional viscous and gravity forces. This was shown to control the drainage and thinning within the middle lamella section of the film. (Figs. 2(a,b) and Region II in Appendix B 2). The balance of surface tension forces and gravity resulted in the development of a plateau border (or a capillary-static meniscus) region near the top support, (Figs. 2( $a, b)$ and Region I in Appendix B 2), while the liquid collects near the bottom support, forming a pendant drop region. (Figs. 2(a,b) and Region III in Appendix B 2). The middle lamella region is shown to connect to the upper and lower regions via short-lengthscale transition regions, where extensional viscous, surface tension and gravity all balance to control the flux out or into the middle region. (Figs. 3(a-f) and Regions A and B in Appendix B 2).
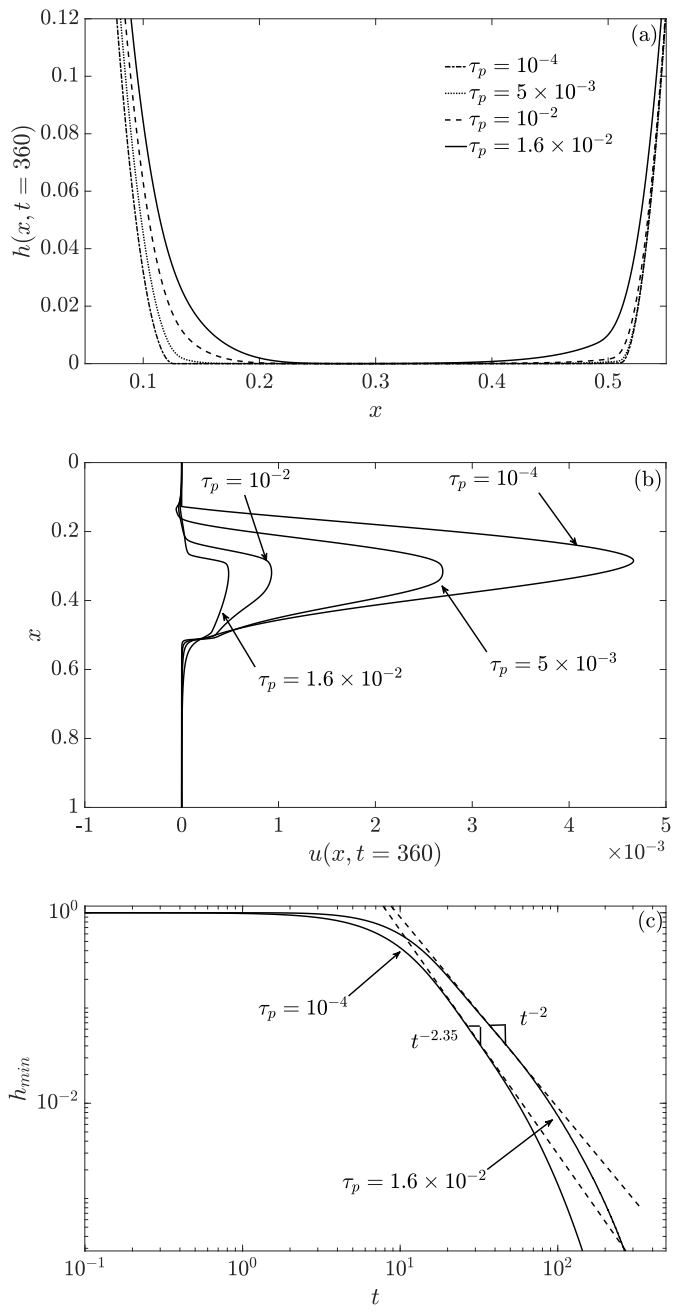

FIG. 16: $(a)$ Film thickness $h(x, t=360)$, and $(b)$ extensional flow speed $u(x, t=360)$, and $(c)$ the global minimum $h_{\min }$ as a function of time $t$, for varying yield stress $\tau_{p}=1.6 \times 10^{-2}, 10^{-2}, 5 \times 10^{-3}, 10^{-4}$, for $n=1.2$, $K_{0}=1, C a=10^{3}$ and $R e=0$. The corresponding thinning rates are $t^{-2.35}$ for $\tau_{p}=10^{-4}$ and $t^{-2}$ for

$$
\tau_{p}=1.6 \times 10^{-2} .
$$

Our model incorporated the effects due to nonNewtonian and viscoplastic behaviour (shear-thinning and yield stress). A variety of non-Newtonian and viscoplastic constitutive relationships, such as Powerlaw, Carreau and Herschel-Bulkley, were investigated to model the apparent viscosity and the yield stress. A study of the system parameters, mainly, the liquid consistency index $K$, the power-law index, $n$, and the liquid yield stress, $\tau_{p}$, was undertaken. Numerical simulations revealed the influence of varying these parameters on the evolution of the film's free surface, its long-time thinning rate and the draining of liquid from the film. For a Newtonian liquid film, we observed power-law behaviour in the thinning rate of the middle lamella section of the film, which is estimated as $t^{-2.25}$ (Fig. 9(c)). This is in com- 
parison to previous studies by Breward ${ }^{23,24}$ and Brush \& Davis $^{25}$ who derive a $t^{-2}$ power-law behaviour. The new and slightly accelerated thinning rate reported here is due to the additional influence of gravity (and possibly contribution from the unsteady flow in the transition regions), which was not considered in these studies. This suggests the long-lived influence of gravity in enhancing the draining of the lamella, and accelerating its thinning rate, hence needs to be included in liquid foam models. Our predictions of power-law thinning behaviour is in contrast to a similar study by Davis et al. ${ }^{27}$. They demonstrated that the lamella thinned non-uniformly with exponential decay of the minimum film thickness, which is significantly faster than the $t^{-2}$ power-law thinning predicted when gravitational effects are negligible ${ }^{23-25}$, and the $t^{-2.25}$ predicted here. While we have not made a direct comparison to determine why this is the case, a possible explanation may be due to their starting film thickness being much smaller than ours. Therefore, their late-time behaviour corresponds to our early-time solution behaviour described in Appendix B 1) - there is a close resemblance in the form of both solutions. This would need to be further investigated before any conclusion is reached.

For a non-Newtonian liquid film, we observed dependence of the power-law index $n$ on the power-law thinning rate, with $t^{-2}$ thinning rate for $n<1$ and $t^{-2.25}$ for $n \geq 1$ (Fig. 9(c)). A similar study done by Brush \& Roper $^{28}$ without the influence of gravity analytically predicted the film's thinning rate to be $t^{-2}$ and independent of the Power-law exponent $n$, which is the same as that for a Newtonian liquid. They concluded that the effect of $n$ was to shift the $h_{\min }$ versus $t$ curve, without affecting the thinning rate. Our numerical results shown in Fig. $9(c)$ are broadly similar to theirs, except for the dependence of the thinning rate on $n$. This weak dependence on shear thinning is a consequence of including gravity. We were unable to validate our predictions using the latetime asymptotic framework described in Appendix B 2 due to not being able to solve the equations analytically, although the asymptotic framework could be extended for a generalised Newtonian liquid (not shown here).

The draining and thinning behaviour of a viscoplastic liquid film was close to that of a Newtonian film for small values of the yield stress $\tau_{p}$. When $\tau_{p}$ was increased, the flow exhibited weakly yielding behaviour primarily in a region around the maximum in the extensional flow (corresponding to the minimum in the film thickness); the width of this weakly yielding region increased as the value of $\tau_{p}$ increases. The weakly yielding region slowed down the extensional flow speed in the lamellar region leading to a relatively thicker middle section in comparison to a film with zero yield stress at any given instant of time. We observed a noticeable decrease in the film's thinning rate as $\tau_{p}$ was increased, with a $t^{-2}$ thinning rate for $n>1$ (in comparison to $t^{-2.35}$ for $\tau_{p}=0$ ) and $t^{-1.7}$ thinning rate for $n=1$ (in comparison to $t^{-2.25}$ for $\left.\tau_{p}=0\right)$. For $n<1$, the dependence was weak with $t^{-1.8}$ thinning rate (in comparison to $t^{-2}$ for $\tau_{p}=0$ ). To the best of our knowledge, the influence of viscoplastic effects due to a yield stress have not been previously investigated in thin liquid film draining flows associated with metallic and polymeric melts using this flow configuration. Our new findings provide the basis to advance the current understanding to a wider class of liquid film draining flows related to foams.

It is known that Power-law fluids are inappropriate for certain low-shear rate flows ${ }^{29}$. Therefore, in the absence of any experimental evidence, our thinning rate predictions for the low shear rates are questionable. Our results identified the parameter, $K=\left(K^{\star} / \mu_{0}^{\star}\right)\left(U^{\star} / L^{\star}\right)^{n-1}$ (a dimensionless liquid consistency index), to play a key role in the draining and thinning dynamics. Decreasing $K$ for fixed $n$ increased the extensional flow speed and corresponding shear rates (which scaled like $1 / K^{1 / n}$, resulting in the film thinning very rapidly on a timescale which scaled like $K^{1 / n}$. Moreover, this also indicated that for small values of $K$, the highest shear rates and severest thinning rates would be for $n<1$. For large values of $K$, the film drains and thins faster for $n>1$ compared to $n<1$. Experimental data for polymeric fluids show that $K \approx\left(10^{-2}-20\right)$ (see Table 1 in Myers $^{29}$ ). For the smaller range of values of $K$, the shear rate would be significantly larger and in the range of validity of the Power-law model for the film thinning rate predictions to be more reliable. However, for these values of $K$ the thinning of the film is very rapid for any power-law behaviour to be observed.

We also investigated the Carreau model which is well behaved at low shear rates ${ }^{29}$. We had to choose a large value of $\lambda^{\star} \approx 10$ s (dimensionless $\lambda=100$; although not unrealistic, e.g., experimental data for polystryrene liquid showed that $\lambda^{\star} \approx 46^{29}$ ) to observe variation in the viscosity from the Newtonian case. We observed thet the film drains and thins more rapidly for $n<1$, compared to $n>1$, unlike the Power-law model (compare Figs. 9 and 12 for Power-law and Carreau models, respectively). The thinning rates appeared to be less dependent on $n$, with a thinning rate estimated to be $t^{-2.25}$, similar to the Newtonian case. The disparity in the results between the Power-law models and the Carreau model highlights the inappropriateness of applying the Powerlaw fluid model at low shear rates. At higher shear rates, one would need to compare both models by choosing appropriate values for $K$ and $\lambda$. Such comparisons can be made, for example, in the shear thinning region by choosing $K^{\star}=\mu_{0}^{\star} \lambda^{\star^{n-1}}$ (or dimensionless $\left.K=\lambda^{n-1}\right)^{28,29}$. Fig. $17(a, b)$ shows $h_{\min }$ versus $t$ for 2 different liquids, Polystyrene and Hydroxylethycellulose, respectively, using the Power-law and Carreau models. The parameter values used are based on those reported for these liquids in Table 1 in Myers $^{29}$, except the value of $n=0.8$ is chosen for both liquids, while their reported values are $n=0.4,0.5$ for Polystyrene and Hydroxylethycellulose, respectively ${ }^{29}$. We were unable to compute the solutions for low values of $n$. We note here that the comparison 

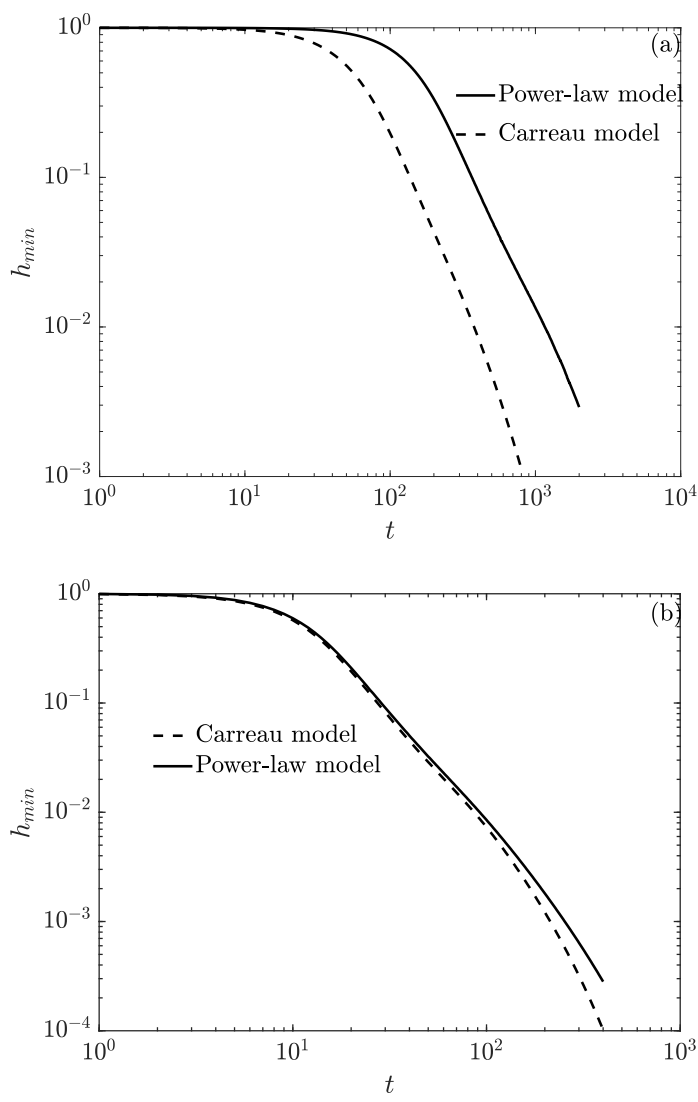

FIG. 17: Comparison between the Power Law and Carreau models for two different liquids, $(a)$ Polystyrene $(n, K, \lambda)=(0.8,4,0.001)$, and $(b)$ Hydroxylethycellulose $(n, K, \lambda)=(0.8,0.51,30)$.

for Polystryene was done using experimentally reported values for $K$ and $\lambda$, while these values for Hydroxylethycellulose were obtained by comparing the two models in the shear thinning region, as explained above. We observe from Fig. 17(a), that the comparison is not good for Polystyrene, but the two models are in good agreement for Hydroxylethycellulose. This is consistent with the results shown in $\S I I I$. The Power-law model predicts low shear rates for large values of $K$ resulting in a large effective viscosity and slower thinning of the film. For smaller values of $K$, even though the shear rates could be low, this is offset by the lower value of $K$, resulting in a lower effective viscosity and quicker thinning of the film.

The theoretical framework developed here is versatile and can be readily adapted to accommodate other complex liquids, such as viscoelastic liquid films. Indeed, one can use the form of the evolution equations written in terms of the extensional stresses, $\tau_{x x}, \tau_{z z}$ and the shear stress $\tau_{x z}$, and use a constitutive model appropriate for a viscoelastic liquid, e.g., Oldroyd-B model ${ }^{32,33}$ to relate these stresses to their corresponding shear rates. This will be investigated in future. Our study is under isother- mal conditions. In molten films, for example, the liquid melt properties (viscosity, surface tension, yield stress and power-law index) could be strongly temperature dependent, especially when they undergo cooling. As part of future work, we would need to extend our model to include temperature-dependent effects. These are relevant in the manufacturing of solid foams by cooling polymeric melts ${ }^{1}$.

In conclusion, the theoretical model developed is versatile in investigating a broad class of non-Newtonian draining flows applicable to a wide range of foam applications. This is both novel and timely and will pioneer further theoretical studies in this area. This insight would form the basis for future developments of this model to incorporate additional effects mentioned above and to utilize the results to investigate the overall behaviour of a foam network, using the framework proposed by Stewart et $a l^{34}$, for example.

\section{ACKNOWLEDGMENTS}

This work was a part of Hani Alahmadi's PhD research at Keele University. Hani gratefully acknowledges financial support from the Ministry of Education, Kingdom of Saudi Arabia, the Saudi Arabian Cultural Bureau in London (UKSACB) and Jouf University.

\section{AUTHOR DECLARATIONS}

No conflicts of interest: The authors have no conflicts to disclose.

\section{DATA AVAILABILITY STATEMENT}

The data that support the findings of this study are available from the corresponding author upon reasonable request.

\section{Appendix A: Derivation of the master PDEs. in (14)}

We use lubrication approximation exploiting the fact that $\epsilon=\frac{H_{0}^{\star}}{L^{\star}} \ll 1$ and expand each of the unknowns variables $\left(u, w, p, \tau^{x x}, \tau^{z z}, \tau^{x z}, h\right)$ as a power series in $\epsilon^{2}$ of the form:

$$
\begin{aligned}
& \left(u, w, p, \tau^{x x}, \tau^{z z}, \tau^{x z}, h\right)=\left(u, w, p, \tau^{x x}, \tau^{z z}, \tau^{x z}, h\right)_{0}(x, z, t) \\
& +\epsilon^{2}\left(u, w, p, \tau^{x x}, \tau^{z z}, \tau^{x z}, h\right)_{1}(x, z, t)+O\left(\epsilon^{4}\right) .
\end{aligned}
$$


For brevity we will ignore the ${ }^{\star}$ on the variables. Substituting this into Eqs. $(12(a-h))$, we obtain at $O(1)$ :

$$
\begin{aligned}
& u_{0 x}+w_{0 z}=0, \\
& \tau_{0 z}^{x z}=0, \\
& -p_{0 z}+\tau_{0 x}^{x z}+\tau_{0 z}^{z z}=0, \\
& w_{0}=u_{0 z}=\tau_{0}^{x z}=0, \text { at } z=0, \\
& -p_{0}+\tau_{0}^{z z}-2 h_{0 x} \tau_{0}^{x z}=\frac{1}{C a} \frac{h_{0 x x}}{\left(1+\epsilon^{2} h_{0 x}^{2}\right)^{3 / 2}}, \text { at } z=h_{0} \\
& \tau_{0}^{x z}=0, \text { at } z=h_{0} .
\end{aligned}
$$

Eqs. (A3), (A5) and (A7) imply that

$$
\tau_{0}^{x z}(x, z, t)=0 .
$$

Integrating Eq. (A4) with respect to $z$ and using Eq. (A5) and (A6), we obtain

$$
p_{0}=\tau_{0}^{z z}-\frac{1}{C a} h_{0 x x} .
$$

To determine $\tau_{0}^{x x, z z}$, we need to analyse the $O\left(\epsilon^{2}\right)$ equations. Before we do this, we note the following: $u_{0 z}=0$, so $u_{0}=u_{0}(x, t)$, using $\tau_{0}^{x z}=0$ and Eq. (12d) at leading order. In addition, $\tau_{0}^{z z}=-\tau_{0}^{x x}$, using Eq. (A2) in Eq. (12d). Eq. (A2) also gives $w_{0 z}=-u_{0 x}$, which on integrating with respect to $z$ and using $w_{0}=0$ at $z=0$, gives $w_{0}(x, z, t)=-u_{0 x} z$. At $O\left(\epsilon^{2}\right)$, we have

$$
\begin{aligned}
& \operatorname{Re}\left(u_{0 t}+u_{0} u_{0 x}+w_{0} u_{0 z}\right)=-p_{0 x}+\tau_{0 x}^{x x}+\tau_{1 z}^{x z}+1, \\
& \operatorname{Re}\left(w_{0 t}+u_{0} w_{0 x}+w_{0} w_{0 z}\right)=-p_{1 z}+\tau_{1 x}^{x z}+\tau_{1 z}^{z z}, \\
& w_{1}=u_{1 z}=\tau_{1 z}^{x z}=0, \text { at } z=0, \\
& \tau_{1}^{x z}+h_{0 x}^{2} \tau_{0}^{x z}+h_{0 x}\left(\tau_{0}^{z z}-\tau_{0}^{x x}\right)=0, \text { at } z=h_{0} .
\end{aligned}
$$

Integrating Eq. (A10) with respect to $z$ and using Eq. (A12), we obtain

$$
\tau_{1}^{x z}=-\left[2 \tau_{0 x}^{x x}+\frac{1}{C a} h_{0 x x x}+1-\operatorname{Re}\left(u_{0 t}+u_{0} u_{0 x}\right)\right] z .
$$

Substituting this into Eq. (A13) gives

$\left.2\left(h_{0} \tau_{0}^{x x}\right)_{x}+h_{0}\left[\frac{1}{C a} h_{0 x x x}+1\right)\right]-\operatorname{Re} h_{0}\left(u_{0 t}+u_{0} u_{0 x}\right)=0$.

Eq. (A15) represents the force balance at the free surface of the extensional stress (represented by the first term), surface tension (represented by the second term), gravity (represented by the third term) and inertia (represented by the last term).

To determine the evolution equation of $h_{0}$ using Eq. $(12 \mathrm{~h})$, we also need to determine $u_{0}$ and the $O\left(\epsilon^{2}\right)$ correction $u_{1}$. We use the constitutive law to determine these. From Eq. (12d), we obtain

$$
\begin{aligned}
& u_{0 x}=\frac{1}{2 \mu\left(\dot{\gamma}_{0}\right)} \tau_{0}^{x x} \\
& u_{1 z}+w_{0 x}=\frac{1}{\mu\left(\dot{\gamma}_{0}\right)} \tau_{1}^{x z}, \Rightarrow \\
& u_{1 z}=\frac{1}{\mu\left(\dot{\gamma}_{0}\right)} \tau_{1}^{x z}-w_{0 x}=\frac{1}{\mu\left(\dot{\gamma}_{0}\right)} \tau_{1}^{x z}+u_{0 x x} z
\end{aligned}
$$

where $\dot{\gamma}_{0}=\left|u_{0 x}\right|$, is the leading order second invariant of the shear rate tensor. Integrating Eq. (A14) and using the boundary condition $u_{1}=0$ at $z=h_{0}$ gives

$$
\begin{aligned}
& u_{1}(x, z, t)=\frac{1}{2}\left[-\frac{1}{\mu\left(\left|u_{0 x}\right|\right)}\left(2 \tau_{0 x}^{x x}+\frac{1}{C a} h_{0 x x x}+1\right.\right. \\
& \left.\left.-\operatorname{Re}\left(u_{0 t}+u_{0} u_{0 x}\right)\right)+u_{0 x x}\right]\left(z^{2}-h_{0}^{2}\right) .
\end{aligned}
$$

Finally, the evolution equation for $h_{0}$ can be obtained from Eq. (12h) as:

$$
\begin{aligned}
& h_{0 t}+Q_{0 x}+\epsilon^{2} Q_{1 x}=0, \\
& Q_{0}=u_{0} h_{0}, \\
& Q_{1}=\frac{1}{3} h_{0}^{3} \frac{1}{\mu\left(\left|u_{0 x}\right|\right)}\left(2 \tau_{0 x}^{x x}+\frac{1}{C a} h_{0 x x x}+1\right. \\
& \left.\left.-\operatorname{Re}\left(u_{0 t}+u_{0} u_{0 x}\right)\right)-u_{0 x x}\right] .
\end{aligned}
$$

Hence, Eqs. (A19),(A15) and (A16) provide a coupled system of three PDEs for the film's free surface evolution, $h_{0}(x, t)$, the extensional stress, $\tau_{0}^{x x}(x, t)$ and the extensional flow speed $u_{0}(x, t)$, respectively. In practise, it is instructive to combine Eqs. (A15) and (A16) to write a single evolution equation for $u_{0}$. This can be written as:

$$
-\operatorname{Re} h\left(u_{0 t}+u_{0} u_{0 x}\right)+4\left(h_{0} \mu\left(\left|u_{0 x}\right|\right) u_{0 x}\right)_{x}+h_{0}\left[\frac{1}{C a} h_{0 x x x}+1\right]=0 .
$$

\section{Appendix B: Asymptotic model}

\section{Early-time evolution}

At early times, the majority of the film exhibits a characteristic concave-out form. This is due to the dominating extensional viscous flow, with the effect of surface tension confined to boundary layers near the ends (see Fig. $2(a, b))$. The early-time similarity solution was identified by Schwartz \& Roy ${ }^{16}$.

The early-time similarity solution for this section of the film is determined primarily by the simplified equations for the film thickness, $h$, and extensional flow speed, $u$, namely

$$
\begin{aligned}
h_{t}+(u h)_{x} & =0, \\
\left(h u_{x}\right)_{x} & =-\frac{1}{4} h .
\end{aligned}
$$


Integrating (B2) with respect to $x$, we obtain

$$
\left.h u_{x}\right|_{x_{1}(t)} ^{x}=h u_{x}=-\frac{1}{4} \int_{x_{1}(t)}^{x} h d x,
$$

where $x_{1}(t)$ is the location at which $u_{x}\left(x_{1}(t)\right)=0$. Applying the method of characteristics to (B1), we obtain

$$
\begin{aligned}
& \frac{d h}{d t}=-u_{x} h=\frac{1}{4} \int_{x_{1}(t)}^{x} h d x, \\
& \frac{d x}{d t}=u(x, t),
\end{aligned}
$$

along the characteristics given by $x=x(\xi, t)$.

Conservation of liquid mass along the characteristics (which is valid at least at early times), implies $h d x=$ $h_{0} d \xi$, where $h_{0}=h(\xi, t=0)$. We take $h_{0}$ equal to a constant which in our simulations is $h_{0}=1$. Using this, we have $\int_{x_{1}(t)}^{x} h d x=h_{0} \int_{\xi_{1}(t)}^{\xi} d \xi=h_{0}\left(\xi-\xi_{1}(t)\right)$, where $x_{1}(t)=x\left(\xi_{1}(t)\right)$. Hence,

$$
\frac{d h}{d t}=\frac{1}{4} h_{0}\left(\xi-\xi_{1}(t)\right) .
$$

Integrating with respect to $t$ and using the initial condition, $h(\xi, t=0)=h_{0}$, we obtain

$$
h(x(\xi, t), t)=h_{0}\left[1+\frac{1}{4}\left(\xi t-\int_{0}^{t} \xi_{1} d t\right)\right] .
$$

Now, we have $h(x(\xi, t), t)=h_{0} \frac{d \xi}{d x}$ (using mass conservation along a characteristic). Hence,

$$
\frac{d \xi}{d x}=1+\frac{1}{4}\left(\xi t-\int_{0}^{t} \xi_{1} d t\right), \quad \text { or } \frac{d \xi}{d x}-\frac{1}{4} \xi t=1-\frac{1}{4} \int_{0}^{t} \xi_{1} d t .
$$

The solution for this using the boundary condition, $x(\xi=$ $0, t)=0($ or $\xi(x=0, t)=0)$, is

$$
\xi(x, t)=-4 \frac{\left[1-e^{x t / 4}\right]}{t}\left[1-\frac{1}{4} \int_{0}^{t} \xi_{1} d t\right] .
$$

Using this, (B7) can be written as

$$
h(x(\xi, t), t)=h_{0} e^{x t / 4}\left[1-\frac{1}{4} \int_{0}^{t} \xi_{1} d t\right] .
$$

Using (B3) and the above relations, we obtain

$$
u_{x}=-\frac{\left[1-e^{-x t / 4}\right]}{t} .
$$

Integrating this and using the boundary condition, $u(x=$ $0, t)=0$, gives

$$
u(x, t)=-\frac{x}{t}-\frac{4}{t^{2}}\left[e^{\left(x_{1}-x\right) t / 4}-e^{x_{1} t / 4}\right] .
$$

Using the boundary condition, $x(\xi=1, t)=1$ (or $\xi(x=$ $1, t)=1$ ), in (B9) gives

$$
1-\frac{1}{4} \int_{0}^{t} \xi_{1} d t=\frac{1}{4} \frac{t}{\left[e^{t / 4}-1\right]} .
$$

Hence,

$$
h(x(\xi, t), t)=\frac{1}{4} h_{0} \frac{t e^{x t / 4}}{\left[e^{t / 4}-1\right]} .
$$

Using the boundary condition, $u(x=1, t)=0$, we can solve for $x_{1}(t)$ as:

$$
x_{1}(t)=\frac{4}{t} \log \left(\frac{t}{4\left[1-e^{-t / 4}\right]}\right) .
$$

\section{Late-time evolution}

Based on the observation from the numerical solutions shown previously for capillary number, $\mathrm{Ca} \gg 1$, we postulate a self-similar structure of the late-time evolution which can be divided into 5 regions (see Fig. 18), namely,

(i) Region I: a quasi-static capillary meniscus in $0 \leq$ $x \leq x_{1}(t)$ where gravity and surface tension forces balance.

(ii) Region II: an almost flat draining section of the film between $x_{1}(t) \leq x \leq x_{2}(t)$, where gravity and extensional viscous forces balance, and surface tension is negligible.

(iii) Region III: a quasi-static pendant drop in $x_{2}(t) \leq$ $x \leq 1$ where gravity and surface tension forces balance.

(iv) Region A: a short transition region of width $O(1 / \mathrm{Ca})$ between regions I and II, where extensional viscous and surface tension forces balance.

(iv) Region B: a short transition region of width $O(1 / \mathrm{Ca})$ between regions II and III where extensional viscous and surface tension forces balance.

The film evolution process is characterised by the key variables: $x_{1}(t)$, representing the location of the leading edge of the capillary meniscus in Region I; $h_{1}(t)$ the film thickness at $x_{1}(t) ; x_{2}(t)$, representing the location of the leading edge of the pendant drop in Region III; $h_{2}(t)$ the film thickness at $x_{2}(t)$, and $h_{\min }(t)$, the minimum film thickness in Region II. We also define $x_{\{1,2\}_{1}}(t)$, representing the location corresponding to the minimum and maximum in the change in curvature $h_{x x x}(t)$ in the transition regions $A$ and $B$, respectively, and $x_{\{1,2\}_{\infty}}=\lim _{t \rightarrow \infty} x_{\{1,2\}_{1}}$ to be their limiting values. The quantities $x_{\{1,2\}_{\infty}}$ characterise the final state (say) separating Region I, with volume $V_{1}$, and Region III, with 


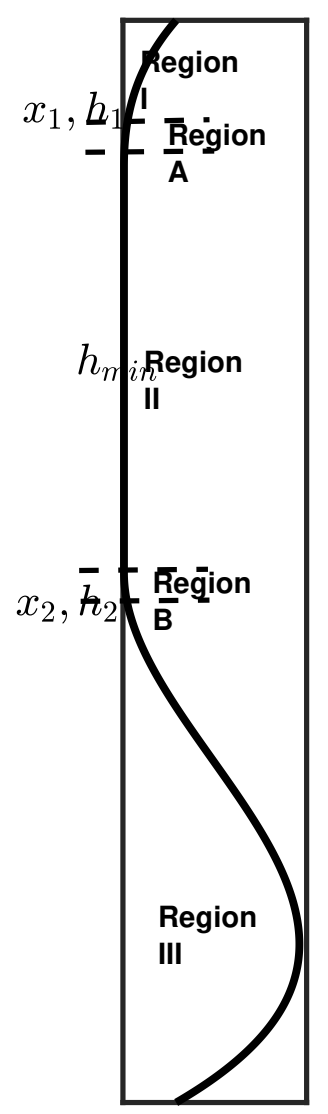

FIG. 18: Late-time self-similar spatial structure of the film thickness $h$, showing Region I, the capillary meniscus, Region II, the almost flat draining section,

Region III, the pendant drop and two transition regions, $\mathrm{A}$ and $\mathrm{B}$. The characteristic variables, $x_{1,2}(t)$, $h_{1,2}$ and $h_{\min }(t)$ are explained in the sections describing each region.

volume $V_{2}=1-V_{1}$ by a film of negligible thickness in Region II. We will show below that the closure conditions, that will enable us to fully describe the asymptotics of the evolution, assumes that the locus of points $\left(x_{1,2}, h_{1,2}\right)$ will lie along the final state of Regions I and III, respectively. Their evolution will be determined by the change in volume in each of these regions due to flux entering or leaving the thin film in Region II.

Figure 19 (solid lines) shows the time evolution of $x_{1,2}$ and $x_{\{1,2\}_{1}}$ (Fig. $19(a)$ ), and $h_{1,2, \min }$ (Fig. $\left.19(b)\right)$ determined from the numerical solution of the PDEs for time $t=5 \times 10^{2}-10^{3}$, with $C a=10^{3}$ and $R e=0$. These are computed from the numerical solution as follows: $x_{1}$ is the location corresponding to the endpoint of the interval near the top of the film where $h_{x x x}+C a$ differs from zero for the first time $\left(>10^{-6}\right.$, in practice; these quantities are necessarily equal to zero for $x<x_{1}$ and non-zero for $x>x_{1}$ ), with corresponding film thickness $h_{1} ; x_{2}$ is the location corresponding to the starting point of the interval near the bottom of the film where $h_{x x x}+C a$ is equal to zero for the first time $\left(<10^{-6}\right.$, in practice; these quantities are necessarily equal to zero for $x>x_{2}$ and non-zero for $x<x_{2}$ ), with corresponding film thickness $h_{2} ; x_{1_{1}}$ is the location corresponding to the minimum value of $h_{x x x}$ near the top of the film where $h_{x x x}<0$ (note that $x_{1}<x_{1_{1}}$ ); $x_{2_{1}}$ is the location corresponding to the maximum value of $h_{x x x}$ near the bottom of the film where $h_{x x x}>0$ (note that $x_{2}>x_{2_{1}}$ ); $h_{\min }$ is the minimum film thickness in Region II, corresponding to the location where the extensional flow speed $u$ is maximum.

We note from Fig. $19(a)$ that $x_{\{1,2\}_{1}}$ are almost constant in time, with $x_{1_{\infty}}=\lim _{t \rightarrow \infty} x_{1} \approx 0.13464$ (corresponding to $V_{1} \approx 0.04$ ) and $x_{2_{\infty}}=\lim _{t \rightarrow \infty} x_{2} \approx 0.511$ (corresponding to $V_{2} \approx 0.96$ ). The evolution of the characteristic variables shown in Fig. 19(a) suggests that the late-time evolution is characterised by two quasi-static regions, regions I and III, and region II connects onto these two regions via a family of solutions representing the transition regions $\mathrm{A}$ and $\mathrm{B}$. The family of solutions in the transition regions emanate from the two quasi-static regions at $x_{1,2}(t)$. Figure $19(b)$ shows that the late-time thinning is severest in the film's middle section followed by less severe thinning near the top and then near the bottom.

We now describe each region in turn.

\section{Region I: the capillary meniscus}

This region lies between $0 \leq x \leq x_{1}$, and is quasi static with $u=0$. The evolution is determined by balancing gravity and surface tension.

$$
h_{x x x}=-C a \text {. }
$$

Integrating thrice with respect to $x$ and applying the boundary condition, $h(x=0)=1$, the solution is given by

$$
h(x)=-\frac{C a}{6} x^{3}+\frac{C x^{2}}{2}+D x+1,
$$

where $C$ and $D$ are constants of integration that need to be determined. To determine $C$ and $D$ we assume that the long-time behaviour of the meniscus is such that it meets the centreline $z=0$ at the location $x=x_{1_{\infty}}$ with zero effective contact angle, i.e., with zero slope, $h_{x}=$ 0 . So, we prescribe $h\left(x_{1_{\infty}}\right)=h_{x}\left(x_{1_{\infty}}\right)=0$. Applying these two boundary conditions at $x=x_{1_{\infty}}$ gives two simultaneous equations for $C$ and $D$, namely,

$$
\begin{gathered}
h\left(x=x_{1_{\infty}}\right)=-\frac{C a}{6} x_{1_{\infty}}^{3}+\frac{C x_{1_{\infty}}^{2}}{2}+D x_{1_{\infty}}+1=0, \\
h_{x}\left(x=x_{1_{\infty}}\right)=-\frac{C a}{2} x_{1_{\infty}}^{2}+C x_{1_{\infty}}+D=0 .
\end{gathered}
$$



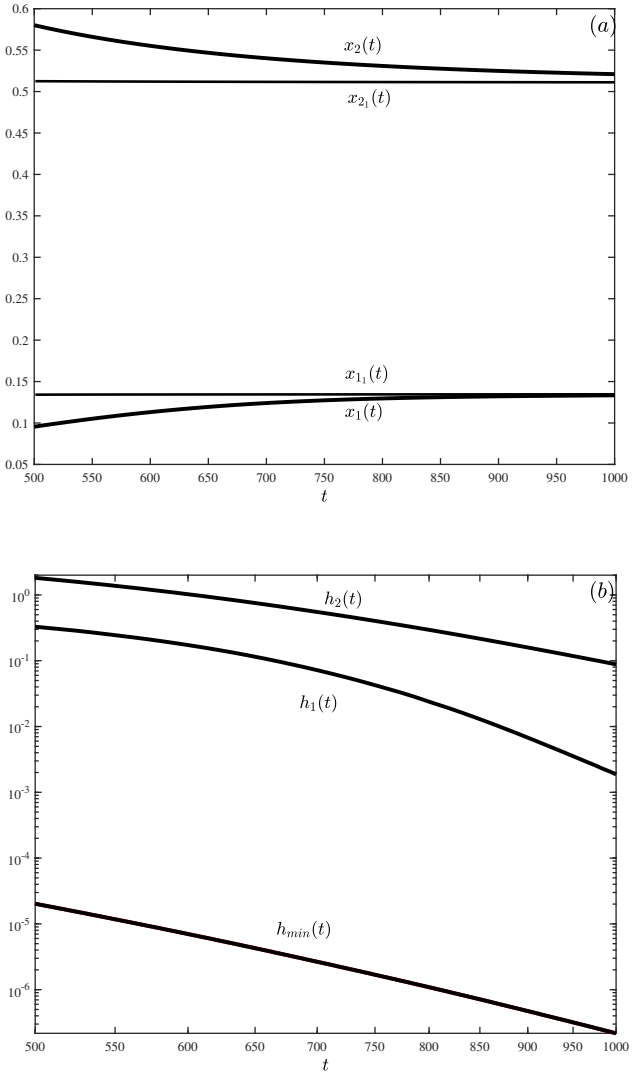

FIG. 19: Evolution of the characteristic variables: $(a)$ $x_{1,2}$ and $x_{\{1,2\}_{1}}$, and $(b) h_{1,2}$ and $h_{m i n}$, for time $t=5 \times 10^{2}-10^{3}$ from the numerical solution (solid curves) and the asymptotic model (dashed curves). The parameter values are $C a=10^{3}$ and $R e=0$.

Solving the above equations simultaneously, we obtain

$$
\begin{aligned}
C & =\frac{2}{x_{1_{\infty}}^{2}}+\frac{2 \operatorname{Cax}_{1_{\infty}}}{3}, \\
D & =-\left(\frac{2}{x_{1_{\infty}}}+\frac{\operatorname{Cax}_{1_{\infty}}^{2}}{6}\right) .
\end{aligned}
$$

Hence,

$$
h(x, t)=-\frac{C a}{6} x^{3}+\frac{C x^{2}}{2}+D x+1,
$$

with $C$ and $D$ given by the above expressions depending on the capillary number $C a$ and $x_{1_{\infty}}$. The unknown quantity $x_{1_{\infty}}$ is determined by imposing that the volume of this region is $V_{1}$. Hence, $\int_{0}^{x_{1}} h d x=V_{1}$. Using this, we obtain:

$$
\begin{aligned}
& \operatorname{Cax}_{1_{\infty}}^{4}-8\left[\frac{1}{x_{1_{\infty}}^{2}}+\frac{\operatorname{Cax}_{1_{\infty}}}{3}\right] x_{1_{\infty}}^{3}+ \\
& 12\left[\frac{2}{x_{1_{\infty}}}+\frac{\operatorname{Cax}_{1_{\infty}}^{2}}{6}\right] \operatorname{Cax}_{1_{\infty}}^{2}+24\left(V_{1}-x_{1_{\infty}}\right)=0 .
\end{aligned}
$$

Eq. (B21) is a quartic equation for $x_{1_{\infty}}$ for a given value of $\mathrm{Ca}$ and $V_{1}$.

Figure 20 plots $h$ versus $x-x_{1_{\infty}}$ (black curves) for $t=5 \times 10^{2}-10^{3}$ using the numerical solution shown in Fig. 2(a), for $\mathrm{Ca}=10^{3}$. The dashed line shows the corresponding late-time asymptotic solution given by Eq. (B20), using $V_{1}=0.04$ (corresponding $x_{1_{\infty}}=0.13464$ ). We observe that the asymptotic solution agrees very well with the scaled numerical solution.

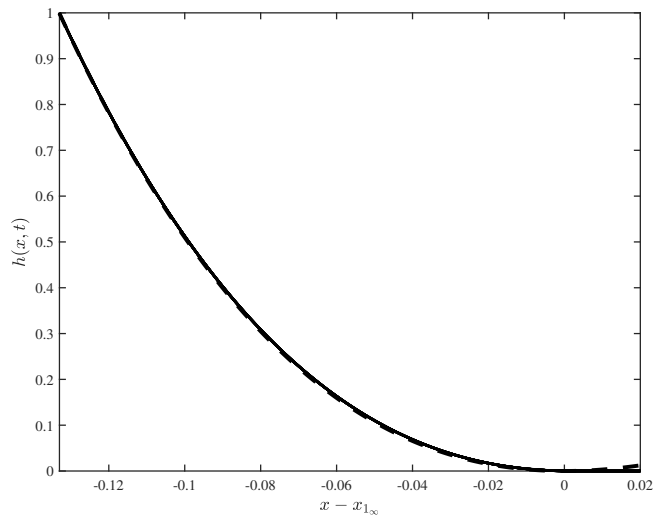

FIG. 20: Evolution of $h$ in region I using data shown in Fig. 2(a) for time $t=5 \times 10^{2}-10^{3}$ (solid black curves) for $C a=10^{3}$. The dashed line shows the corresponding late-time asymptotic solution given by Eq. (B20), using

$$
\left.V_{1}=0.04 \text { (corresponding } x_{1_{\infty}}=0.13464\right) \text {. }
$$

\section{Region II - the middle draining section}

Extensional viscous forces control the film evolution in this region between $x_{1} \leq x \leq x_{2}$. The film is almost flat, so surface tension effects are negligible. The flow in this region is controlled by competing extensional viscous forces and gravity.

$$
\begin{aligned}
h_{t}+(u h)_{x} & =0, \\
\left(h u_{x}\right)_{x} & =-\frac{1}{4} h .
\end{aligned}
$$

Integrating (B22) with respect to $x$, we obtain

$$
(u h)(x, t)=(u h)\left(x_{1}\right)-\int_{x_{1}}^{x} h_{t} d x, \text { or } Q=Q_{1}(t)-\int_{x_{1}}^{x} h_{t} d x
$$

where $Q=u h$ and $Q_{1}(t)=(u h)\left(x=x_{1}(t)\right)$. Hence,

$$
h(x, t)=\frac{Q_{1}(t)-\int_{x_{1}}^{x} h_{t} d x}{u(x, t)} .
$$

Also, using (B24), we can write

$$
Q_{2}(t)=Q_{1}(t)-\int_{x_{1}}^{x_{2}} h_{t} d x
$$


where $Q_{2}(t)=(u h)\left(x=x_{2}(t)\right)$. Using Eq. (B25), we can rewrite Eq. (B23) in terms of $u$ as:

$$
\left(\left[Q_{1}(t)-\int_{x_{1}}^{x} h_{t} d x\right] \frac{u_{x}}{u}\right)_{x}=-\frac{1}{4} \frac{\left[Q_{1}(t)-\int_{x_{1}}^{x} h_{t} d x\right]}{u} .
$$

We are unable to determine the solution of Eqs. (B25,B27) analytically. However, it will be useful to determine the behaviour of $h$ and $u$ near $x_{1,2}$ to match with regions $\mathrm{A}$ and $\mathrm{B}$, respectively. We rewrite Eq. (B27) as

$$
Q(x, t)\left[u u_{x x}-u_{x}^{2}\right]+Q_{x}(x, t) u u_{x}+\frac{1}{4} Q u=0,
$$

where $Q(x, t)$ is defined in Eq. (B24). We seek the behaviour of $u$ subject to the boundary conditions, $u\left(x_{1,2}, t\right)=u_{x}\left(x_{1,2}, t\right)=0$. We expand $u$ in a Taylor's series about $x=x_{1,2}$ :

$$
\begin{aligned}
& u(x, t)=u^{(2)}\left(x_{1,2}, t\right) \frac{\left(x-x_{1,2}\right)^{2}}{2}+u^{(3)}\left(x_{1,2}, t\right) \frac{\left(x-x_{1,2}\right)^{3}}{6}+ \\
& u^{(4)}\left(x_{1,2}, t\right) \frac{\left(x-x_{1,2}\right)^{4}}{24}+\ldots
\end{aligned}
$$

Here the superscript represents a higher order derivative with respect to $x$. We also expand $Q(x, t)$ in a Taylor's series about $x=x_{1,2}$ :

$$
\begin{aligned}
& Q(x, t)=Q_{1,2}(t)+Q^{(1)}\left(x_{1,2}, t\right)\left(x-x_{1,2}\right)+ \\
& Q^{(2)}\left(x_{1,2}, t\right) \frac{\left(x-x_{1,2}\right)^{2}}{2}+\ldots
\end{aligned}
$$

Substituting the above in Eq. (B28), we obtain the following sequence of problems. At $O\left(\left(x-x_{1,2}\right)^{2}\right)$, we have $u^{(2)}\left(x_{1,2}, t\right)=\frac{1}{4}$. At $O\left(\left(x-x_{1,2}\right)^{3}\right)$, we obtain:

$$
\begin{aligned}
& -Q_{1,2} u^{(2)}\left(x_{1,2}\right) u^{(3)}\left(x_{1,2}\right)-Q^{(1)}\left(x_{1,2}, t\right)\left[u^{(2)}\left(x_{1,2}\right)\right]^{2}+ \\
& \frac{2}{3} Q_{1,2} u^{(2)}\left(x_{1,2}\right) u^{(3)}\left(x_{1,2}\right)+Q^{(1)}\left(x_{1,2}, t\right)\left[u^{(2)}\left(x_{1,2}\right)\right]^{2}+ \\
& \frac{1}{24} Q_{1,2} u^{(3)}\left(x_{1,2}\right)+\frac{1}{8} Q^{(1)}\left(x_{1,2}, t\right) u^{(2)}\left(x_{1,2}\right)=0 .
\end{aligned}
$$

Using $u^{(2)}\left(x_{1,2}\right)=1 / 4, \quad=\quad$ we obtain $u^{(3)}\left(x_{1,2}\right)=\frac{3}{4} \frac{Q^{(1)}\left(x_{1,2}, t\right)}{Q_{1,2}}$. The solutions for $u$ and $h$ about $x=x_{1,2}$ are then given by

$$
\begin{aligned}
u(x, t) & =\frac{1}{8}\left[x-x_{1,2}(t)\right]^{2}\left[1+\frac{Q^{(1)}\left(x_{1,2}, t\right)}{Q_{1,2}}\left(x-x_{1,2}\right)\right]+ \\
O & \left(\left(x-x_{1,2}\right)^{4}\right), \\
h(x, t) & =\frac{8 Q_{1,2}(t)}{\left[x-x_{1,2}(t)\right]^{2}},
\end{aligned}
$$

near $x=x_{1,2}, \quad$ respectively. We approximate $Q=Q_{1}(t)-\int_{x_{1}}^{x} h_{t} d x$ near $x=x_{1}$ as
$Q=Q_{1}(t)-h_{1_{t}}(t)\left(x-x_{1}\right)+O\left(\left(x-x_{1}\right)^{2}\right) . \quad$ Hence, $Q^{(1)}\left(x_{1}, t\right) \approx-h_{1_{t}}(t)$, so the solution of $u$ and $h$ near $x=x_{1}$ can be written as

$$
\begin{aligned}
u(x, t) & =\frac{1}{8}\left[x-x_{1}(t)\right]^{2}\left[1-\frac{h_{1_{t}}(t)}{Q_{1}}\left(x-x_{1}\right)\right]+ \\
O & \left(\left(x-x_{1}\right)^{4}\right) \\
h(x, t) & =\frac{8 Q_{1}(t)}{\left[x-x_{1}(t)\right]^{2}}
\end{aligned}
$$

respectively. Similarly, we approximate $Q=Q_{1}(t)-\int_{x_{1}}^{x} h_{t} d x=Q_{2}(t)+\int_{x}^{x_{2}} h_{t} d x$ near $x=x_{2}$ as $Q=Q_{2}(t)-h_{2_{t}}(t)\left(x-x_{2}\right)+O\left(\left(x-x_{2}\right)^{2}\right)$. Hence, $Q^{(1)}\left(x_{2}, t\right) \approx-h_{2_{t}}(t)$, so the solution of $u$ and $h$ near $x=x_{2}$ can be written as

$$
\begin{aligned}
u(x, t) & =\frac{1}{8}\left[x-x_{2}(t)\right]^{2}\left[1-\frac{h_{2_{t}}(t)}{Q_{2}}\left(x-x_{2}\right)\right]+ \\
O & \left(\left(x-x_{2}\right)^{4}\right), \\
h(x, t) & =\frac{8 Q_{2}(t)}{\left[x-x_{2}(t)\right]^{2}},
\end{aligned}
$$

respectively.

In order to make analytical progress we resort to making some meaningful approximations, particularly, to the unsteady term $h_{t}$. The accuracy of the solution based on these approximations will be tested against corresponding numerical solutions.

Quasi-steady approximation $h_{t} \approx 0\left(Q_{1}=Q_{2}=Q\right)$

Eq. (B27) can then be written as:

$$
\left(\frac{u_{x}}{u}\right)_{x}=-\frac{1}{4 u}, \text { or } u u_{x x}-u_{x}^{2}+\frac{1}{4} u=0,
$$

subject to the boundary conditions, $u\left(x_{1}(t)\right)=$ $u\left(x_{2}(t)\right)=0$. This has solution

$u(x, t)=\left[\frac{\left(x_{2}(t)-x_{1}(t)\right)^{2}}{8 \pi^{2}}\right] \sin ^{2}\left(\pi\left[\frac{x-x_{1}(t)}{x_{2}(t)-x_{1}(t)}\right]\right)$.

Hence, the approximate solution in this region is given by:

$$
\begin{aligned}
& h(x, t)=\left[\frac{8 \pi^{2} Q(t)}{\left(x_{2}(t)-x_{1}(t)\right)^{2}}\right] \frac{1}{\sin ^{2}\left(\pi\left[\frac{x-x_{1}(t)}{x_{2}(t)-x_{1}(t)}\right]\right)}, \\
& u(x, t)=\left[\frac{\left(x_{2}(t)-x_{1}(t)\right)^{2}}{8 \pi^{2}}\right] \sin ^{2}\left(\pi\left[\frac{x-x_{1}(t)}{x_{2}(t)-x_{1}(t)}\right]\right),
\end{aligned}
$$

where $Q(t)$ is a constant flux in this region. The maximum extensional flow speed, $u_{\max }(t)$ and minimum film thickness, $h_{\min }(t)$, in this region is at $x=x_{1}(t)+\left(x_{2}(t)-\right.$ 
$\left.x_{1}(t)\right) / 2$ and is given by

$$
\begin{aligned}
h_{\text {min }}(t) & =\frac{8 \pi^{2} Q(t)}{\left[x_{2}(t)-x_{1}(t)\right]^{2}}, \\
u_{\max }(t) & =\frac{\left[x_{2}(t)-x_{1}(t)\right]^{2}}{8 \pi^{2}} .
\end{aligned}
$$

The unknown flux $Q(t)$ and the evolution of $x_{1}(t)$ and $x_{2}(t)$ will be determined from the transition regions $A$ and $B$. Figure 21 shows the family of solutions characterised by $x_{1,2}$ for the extensional flow speed $u(x, t)$ versus $\left(x-x_{1}(t)\right) /\left(x_{2}(t)-x_{1}(t)\right)$ for $t=5 \times 10^{2}-10^{3}$ using the numerical solution shown in Fig. 2(a). The dashed curves show particular solutions of Eq. (B40) for $x_{1,2}$ evaluated from the numerical solution at $t=$ $5 \times 10^{2}, 8 \times 10^{2}, 10^{3}$, respectively. We observe that although the quasi-steady approximation captures the general trends, assuming a constant flux of liquid through this region does not work very well in describing its evolution. This suggests the important contribution of the unsteady term $h_{t}$ in this region.

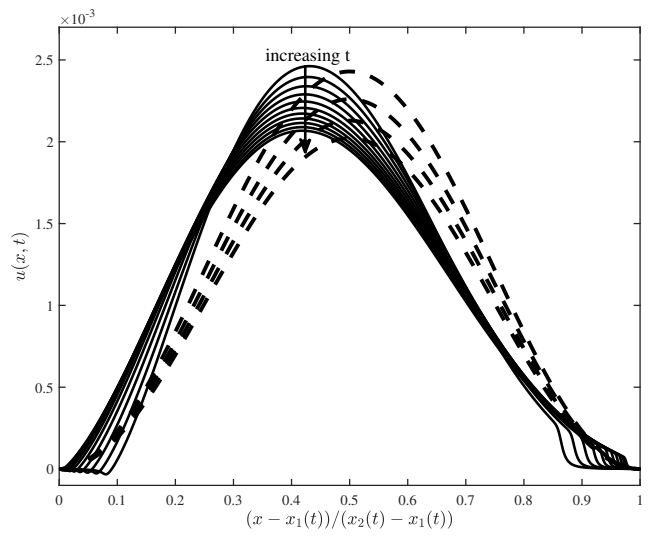

FIG. 21: Evolution of $u$ in region II using data shown in Fig. 2(a) for time $t=5 \times 10^{2}-10^{3}$. The dashed curves show the solution of Eq. (B40) for $x_{1,2}$ evaluated from the numerical solution at $t=5 \times 10^{2}, 8 \times 10^{2}, 10^{3}$, respectively.

\section{Approximation $h_{t} \approx h_{m i n, t}$}

A simple approximation of the unsteady term $h_{t}$ is to assume it to be constant, $h_{t} \approx h_{\min , t}$ (say), where $h_{\min }$ is the minimum film thickness of the middle section of the film. Using this approximation, Eqs. (B25,B27) can be simplified to

$$
\begin{aligned}
& h(x, t)=\frac{Q_{1}(t)-h_{\min , t}\left(x-x_{1}\right)}{u(x, t)}, \\
& \left(\left[Q_{1}(t)-h_{\min , t}\left(x-x_{1}\right)\right] \frac{u_{x}}{u}\right)_{x}= \\
& -\frac{1}{4} \frac{\left[Q_{1}(t)-h_{\min , t}\left(x-x_{1}\right)\right]}{u} .
\end{aligned}
$$

We are unable to solve Eq. (B44) for the solution of $u$ analytically. Using the approximation $h_{t} \approx h_{m i n, t}$ in Eq. (B26), we obtain

$$
\begin{aligned}
Q_{2}(t) & =Q_{1}(t)-h_{\text {min,t }}\left(x_{2}-x_{1}\right), \Rightarrow \\
h_{m i n, t} & =\frac{\left[Q_{1}(t)-Q_{2}(t)\right]}{\left[x_{2}(t)-x_{1}(t)\right]},
\end{aligned}
$$

which provides an evolution equation for $h_{\text {min }}$. Also, using this approximation, $h_{1,2_{t}} \approx h_{\text {min,t }}$, hence $u$ and $h$ near $x=x_{1,2}$ can be written as:

$$
\begin{aligned}
u(x, t) & =\frac{1}{8}\left[x-x_{1}(t)\right]^{2}\left[1-\frac{h_{\text {min }, t}(t)}{Q_{1}}\left(x-x_{1}\right)\right] \\
& +O\left(\left(x-x_{1}\right)^{4}\right), \\
h(x, t) & =\frac{8 Q_{1}(t)}{\left[x-x_{1}(t)\right]^{2}}, \\
u(x, t) & =\frac{1}{8}\left[x-x_{2}(t)\right]^{2}\left[1-\frac{h_{m i n, t}(t)}{Q_{2}}\left(x-x_{2}\right)\right] \\
& +O\left(\left(x-x_{2}\right)^{4}\right), \\
h(x, t) & =\frac{8 Q_{2}(t)}{\left[x-x_{2}(t)\right]^{2}},
\end{aligned}
$$

respectively.

\section{Region III - the pendant drop}

This region lies between $x_{2} \leq x \leq 1$, and is quasi static with $u=0$. The evolution is determined by balancing gravity and surface tension.

$$
h_{x x x}=-C a,
$$

which on integrating thrice with respect to $x$ and using the boundary condition, $h(1)=1$, gives

$$
h(x)=-\frac{C a}{6}(x-1)^{3}+C \frac{(x-1)^{2}}{2}+D(x-1)+1,
$$

where $C$ and $D$ are constants of integration that need to be determined. To determine $C$ and $D$ we assume that the long-time behaviour of the pendant drop is such that it meets the centreline $z=0$ at the location $x=x_{2_{\infty}}$ with zero effective contact angle, i.e., with zero slope, $h_{x}=$ 0 . So, we prescribe $h\left(x_{2_{\infty}}\right)=h_{x}\left(x_{2_{\infty}}\right)=0$. Applying these two boundary conditions at $x=x_{2_{\infty}}$ gives two simultaneous equations for $C$ and $D$, namely,

$$
\begin{aligned}
& C=\frac{2}{\left(x_{2_{\infty}}-1\right)^{2}}+\frac{2 C a\left(x_{2_{\infty}}-1\right)}{3}, \\
& D=-\left(\frac{2}{\left(x_{2_{\infty}}-1\right)}+\frac{C a\left(x_{2_{\infty}}-1\right)^{2}}{6}\right),
\end{aligned}
$$

to give

$$
h(x, t)=-\frac{C a}{6}(x-1)^{3}+\frac{C(x-1)^{2}}{2}+D(x-1)+1,
$$


with $C$ and $D$ given by the above expressions, depending on the capillary number $C a$ and $x_{2_{\infty}}$. The unknown quantity $x_{2_{\infty}}$ is determined by imposing that the volume of this region is $V_{2}=1-V_{1}$. Hence, $\int_{x_{2_{\infty}}}^{1} h d x=V_{2}$. Using this, we obtain:

$C a\left(x_{2_{\infty}}-1\right)^{4}-8\left[\frac{1}{\left(x_{2_{\infty}}-1\right)^{2}}+\frac{C a\left(x_{2_{\infty}}-1\right)}{3}\right]\left(x_{2_{\infty}}-1\right)^{3}$ $+12\left[\frac{2}{\left(x_{2_{\infty}}-1\right)}+\frac{C a\left(x_{2_{\infty}}-1\right)^{2}}{6}\right]\left(x_{2_{\infty}}-1\right)^{2}+$

$24\left(V_{2}-x_{2_{\infty}}+1\right)=0$.

Eq. (B54) is a quartic equation for $x_{2_{\infty}}$ for a given value of $\mathrm{Ca}$ and $\mathrm{V}_{2}$.

Fig. 22 plots $h$ versus $x-x_{2_{\infty}}$ (black curves) for $t=5 \times 10^{2}-10^{3}$ using the numerical solution shown in Fig. 2(a), for $C a=10^{3}$. The dashed line shows the corresponding late-time asymptotic solution given by Eq. (B53), using $V_{2}=1-0.04=0.96$ (corresponding $\left.x_{2_{\infty}}=0.511\right)$. We observe that the asymptotic solution agrees very well with the scaled numerical solution.

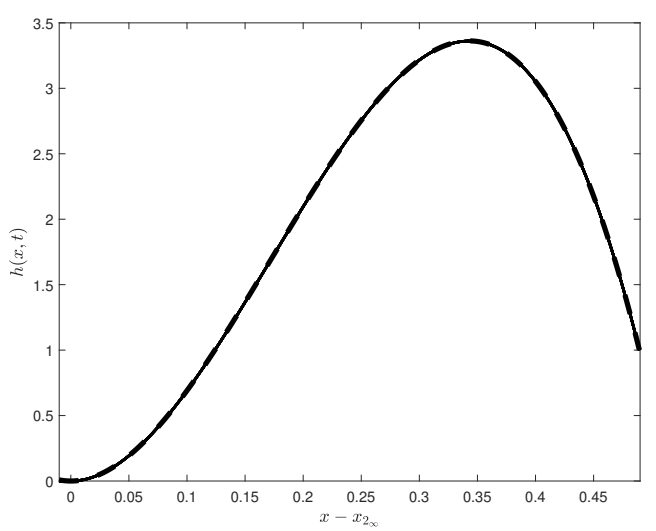

FIG. 22: Evolution of $h$ in region III using data shown in Fig. 2(a) for time $t=5 \times 10^{2}-10^{3}$ (solid black curves) for $C a=10^{3}$. The dashed line shows the corresponding late-time asymptotic solution given by Eq. (B53), using $V_{2}=1-0.04=0.96$ (corresponding

$$
\left.x_{2_{\infty}}=0.511\right) \text {. }
$$

\section{Region $B$ : the transition region between regions $I I$ and $I I I$}

This region around $x=x_{2_{1}}$ is characterised by a large change in the curvature $h_{x x x}$ (see Fig. $3(c)$ ). Hence, surface tension due to changes in curvature is the primary mechanism driving a downward flow into the pendant drop in region III with gravity playing a secondary role further contributing to the downward flow; this flow is resisted by the extensional viscous forces. The lengthscale of this region based on balancing surface tension and extensional viscous forces is of $O(1 / \mathrm{Ca})$. Letting $x=x_{2_{1}}+(1 / C a) \xi$, we can write the evolution equations for $h$ and $u$ as:

$$
\begin{aligned}
& \frac{1}{C a} h_{t}-x_{2_{1_{t}}} h_{\xi}+(u h)_{\xi}=0, \\
& 4\left(h u_{\xi}\right)_{\xi}+h\left(h_{\xi \xi \xi}+\frac{1}{C a^{2}}\right)=0 .
\end{aligned}
$$

For $\mathrm{Ca} \gg 1, \mathrm{Eq}$. (B55) can be written at leading order in $1 / C a$ as: $-x_{2_{1_{t}}} h_{\xi}+(u h)_{\xi}=0$. We assume that any changes to the flux in the moving frame of reference are negligible, hence Eq. (B55) simplifies to $(u h)_{\xi}=0$. Integrating gives $u h=Q_{2}(t)$, where $Q_{2}(t)$ is the flux out of this region into region III. Substituting $u=Q_{2}(t) / h$ into Eq. (B56) gives the following boundary-value problem for $h$ :

$$
-4 Q_{2}(t)\left[\frac{1}{h} h_{\xi}\right]_{\xi}+h\left(h_{\xi \xi \xi}+\frac{1}{C a^{2}}\right)=0
$$

subject to the boundary conditions

$$
\begin{aligned}
& h \rightarrow \frac{8 Q_{2} C a^{2}}{\left(\xi-\xi_{2}\right)^{2}}, \quad \xi \rightarrow-\infty, \quad \xi_{2}=C a\left(x_{2}-x_{2_{1}}\right)>0, \\
& h \rightarrow-\frac{1}{6 C a^{2}}\left(\xi-\xi^{1}\right)^{3}+\frac{C\left(\xi-\xi^{1}\right)^{2}}{2 C a^{2}} \\
& +\frac{D}{C a}\left(\xi-\xi^{1}\right)+1, \quad \xi \rightarrow \infty, \text { where } \xi^{1}=C a\left(1-x_{2_{1}}\right)>0,
\end{aligned}
$$

with $C$ and $D$ defined in region III above. Note that we leave the $\frac{1}{C a^{2}} h$ term in Eq. (B57a) so that we can match with regions II and III. The boundary conditions in Eq. $(\mathrm{B} 57 b, c)$ are obtained by taking the limit as $x \rightarrow x_{2}^{-}$in Eq. (B33) and $x \rightarrow x_{2}^{+}$in Eq. (B53), respectively.

In principle, we can use a shooting method to solve for the eigenvalue $Q_{2}$ in order to determine the numerical solution for the boundary value problem in Eq. (B57). For a given $C a \gg 1, x_{2}, x_{2_{\infty}}$ and $V_{2}$ (these quantities are evaluated at some time $t$ ), we start with the boundary condition at the downstream end, Eq. (B57c) (evaluated at $\xi=\xi_{2}=C a\left(x_{2}-x_{2_{1}}\right)$, where $\xi_{2} \gg 1$ and $\left.\xi_{2}<\xi^{1}\right)$, choosing $Q_{2}$ as the shooting parameter until the boundary condition at the upstream end, Eq. (B57b), is satisfied (evaluated at $\xi=-\xi_{2_{2}}$, where $\xi_{2_{2}} \gg 1$ ). In practice, we shoot with $Q_{2}$ to satisfy $u_{\xi \xi}=Q_{2}\left[2 \frac{h_{\xi}^{2}}{h^{3}}-\frac{h_{\xi \xi}}{h^{2}}\right] \rightarrow \frac{1}{4 C a^{2}}$ as $\xi \rightarrow-\infty$. Fig. $23(a, b)$ compares the computed solution of $h$ and $u$ (solid lines) with the similarity solution given by Eqs. (B55,B57), using the similarity coordinate $\xi$ for $t=$ $(5,6,7,8) \times 10^{2}$. The values of $Q_{2}$ computed using the shooting method are $Q_{2}=(2.70,1.38,0.426,0.174) \times$ $10^{-7}$, for $t=(5,6,7,8) \times 10^{2}$, respectively. We use values of $x_{2}$ computed from the numerical solution shown in Fig. $19(a)$ at the given times, and $x_{2_{1}}(t)=x_{2_{\infty}}=0.510583$. We observe that the similarity solution clearly captures 

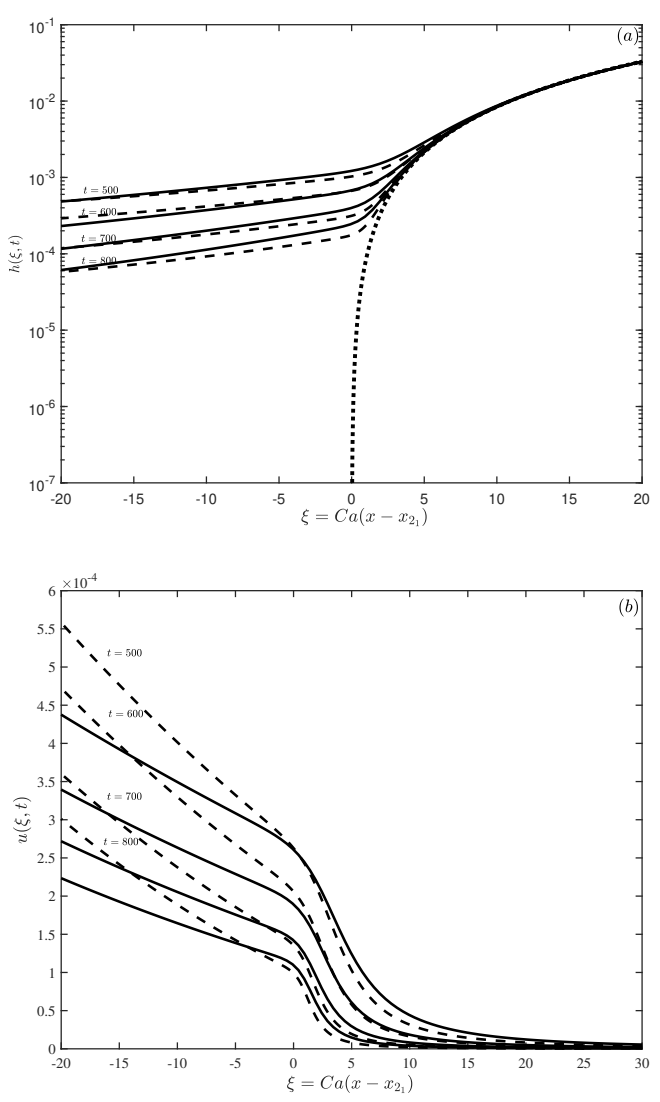

FIG. 23: Computed film thickness profiles, $h,(a)$ plotted on a semi-logarithmic axis and the extensional

flow speed, $u,(b)$ (solid lines) using the similarity coordinate $\xi=C a\left(x-x_{2_{1}}(t)\right)$ for $t=(5,6,7,8) \times 10^{2}$.

The dashed lines show the corresponding late-time similarity solution given by Eqs. (B55,B57). The dotted curve in $(a)$ shows the quasi-static solution of region III given by Eq. (B53) in the similarity variable $\xi$ for $0 \leq \xi \leq \xi^{1}$. The parameter values are $C a=10^{3}, R e=0$ and $x_{2_{\infty}}=0.510583$.

the qualitative characteristics of the quasi-static evolution, namely, the family of solutions of $h$ (see solid lines (numerical solution) and dashed lines (similarity solution) in Fig. 23(a)) emanating from the quasi-static solution of region III given by Eq. (B53) in the similarity variable $\xi$ for $0 \leq \xi \leq \xi^{1}$ (see dotted line in Fig. 23(a)), and the sudden deceleration of the flow $u$ resulting in a shock-like discontinuity near $\xi=0$ (Fig. 23(b)). Although the quantitative match is not perfect, the similarity solution given by Eqs. (B57,B55) captures very well the dynamics in this transition region.

\section{Region $A$ : the transition region between regions $I$ and $I I$}

This region lies around $x_{1_{1}}$, and is also characterised by a large change in the curvature $h_{x x x}$ (see Fig. $3(f)$ ).
Hence, surface tension due to changes in curvature is the primary mechanism driving an upward flow against gravity (see Fig. 2(e) for $u$ in this region). In the upstream part of this region, although, changes in curvature are negligible in comparison to gravity, and the flow changes direction to a downward gravity-driven flow (see Fig. 3(e) for $u$ in the upstream part of this region). Below we describe the evolution of $h$ and $u$ this region based on the above observations. The lengthscale of this region by balancing surface tension and extensional viscous forces is of $O(1 / C a)$. Letting $x=x_{1_{1}}+(1 / C a) \xi$, we can write the evolution equations for $h$ and $u$ as:

$$
\begin{aligned}
& \frac{1}{C a} h_{t}-x_{1_{1_{t}}} h_{\xi}+(u h)_{\xi}=0, \\
& 4\left(h u_{\xi}\right)_{\xi}+h\left(h_{\xi \xi \xi}+\frac{1}{C a^{2}}\right)=0 .
\end{aligned}
$$

For $\mathrm{Ca} \gg 1$, Eq. (B58) at leading order in $O(1 / \mathrm{Ca})$ can be written as: $-x_{1_{1_{t}}} h_{\xi}+(u h)_{\xi}=0$. We assume that any changes to the flux in the moving frame of reference are negligible, hence (B58) simplifies to $(u h)_{\xi}=0$. Integrating gives $u h=-Q_{1}(t)$ for $\xi<0^{-}$and $u h=Q_{1}(t)$ for $\xi>0^{+}$. We assume that the flux changes sign over a much smaller lengthscale, and the flux is of the same magnitude but opposite sign on either side of $\xi=0$. The numerical solutions suggest this, and also that $Q_{1}$ is small $\left(Q_{1} \ll Q_{2}\right)$, so this approximation is reasonable. Using this, we obtain

$$
u=\frac{-Q_{1}}{h}, \text { if } \xi<0 ; u=\frac{Q_{1}}{h}, \text { if } \xi>0 .
$$

Substituting Eq. (B60) into Eq. (B59) gives the following boundary-value problem for $h$ :

$$
-4\left[\mathcal{H}(Q) \frac{1}{h} h_{\xi}\right]_{\xi}+h\left(h_{\xi \xi \xi}+\frac{1}{C a^{2}}\right)=0,
$$

subject to the boundary conditions

$$
\begin{aligned}
& h \rightarrow-\frac{1}{6 C a^{2}}\left(\xi^{1}+\xi\right)^{3}+\frac{C\left(\xi^{1}+\xi\right)^{2}}{2 C a^{2}} \\
& +\frac{D}{C a}\left(\xi^{1}+\xi\right)+1, \quad \xi \rightarrow-\infty, \text { where } \xi^{1}=\operatorname{Cax}_{1_{1}}>0,
\end{aligned}
$$

$$
h \rightarrow \frac{8 Q_{1} C a^{2}}{\left(\xi-\xi_{1}\right)^{2}}, \quad \xi \rightarrow \infty, \quad \xi_{1}=C a\left(x_{1}-x_{1_{1}}\right)<0,
$$

where the piecewise constant function $\mathcal{H}(Q)=-Q_{1}$ if $\xi<0$ and $Q_{1}$ if $\xi>0 ; C$ and $D$ are defined in region I above. The boundary conditions in Eq. (B61b,c) are obtained by taking the limit as $x \rightarrow x_{1}^{-}$in Eq. (B20) and $x \rightarrow x_{1}^{+}$in Eq. (B33), respectively. Similar to region $\mathrm{B}$, we use a shooting method to determine the numerical solution of the boundary value problem in Eq. (B61) using $Q_{1}$ as the shooting parameter. In practice, we use a regularised form of the function $\mathcal{H}=Q_{1} \tanh [A \xi]$, where $A>0(A=1$ in the solutions shown in Fig. 
24). For a given $\mathrm{Ca}, x_{1}, x_{1_{\infty}}$ and $V_{1}$ (these quantities are evaluated at some time $t$ ), we start with the boundary condition at the upstream end, Eq. (B61b) (evaluated at $\xi=\xi_{1}=C a\left(x_{1}-x_{1_{1}}\right)<0$, where $\left|\xi_{1}\right| \gg 1$ and $\left|\xi_{1}\right|<\xi^{1}$ ), choosing $Q_{1}$ as the shooting parameter until the boundary condition at the downstream end, Eq. (B61c), is satisfied (evaluated at $\xi=\xi_{1_{2}}$, where $\left.\xi_{1_{2}} \gg 1\right)$. In practice, we shoot with $Q_{1}$ to satisfy $u_{\xi \xi}=Q_{1}\left[2 \frac{h_{\xi}^{2}}{h^{3}}-\frac{h_{\xi \xi}}{h^{2}}\right] \rightarrow \frac{1}{4 C a^{2}}$ as $\xi \rightarrow \infty$.

Fig. 24 compares the computed solution of $h$ and $u$ (solid lines) with the similarity solution given by Eqs. (B58,B61), using the similarity coordinate $\xi$ for $t=$ $(5,6,7,8) \times 10^{2}$. The values of $Q_{1}$ computed using the shooting method are $Q_{1}=(2,0.05,0.001,0.0001) \times 10^{-9}$, for $t=(5,6,7,8) \times 10^{2}$, respectively (Note that $Q_{2} \ll Q_{1}$, as observed from the numerical solutions). We use values
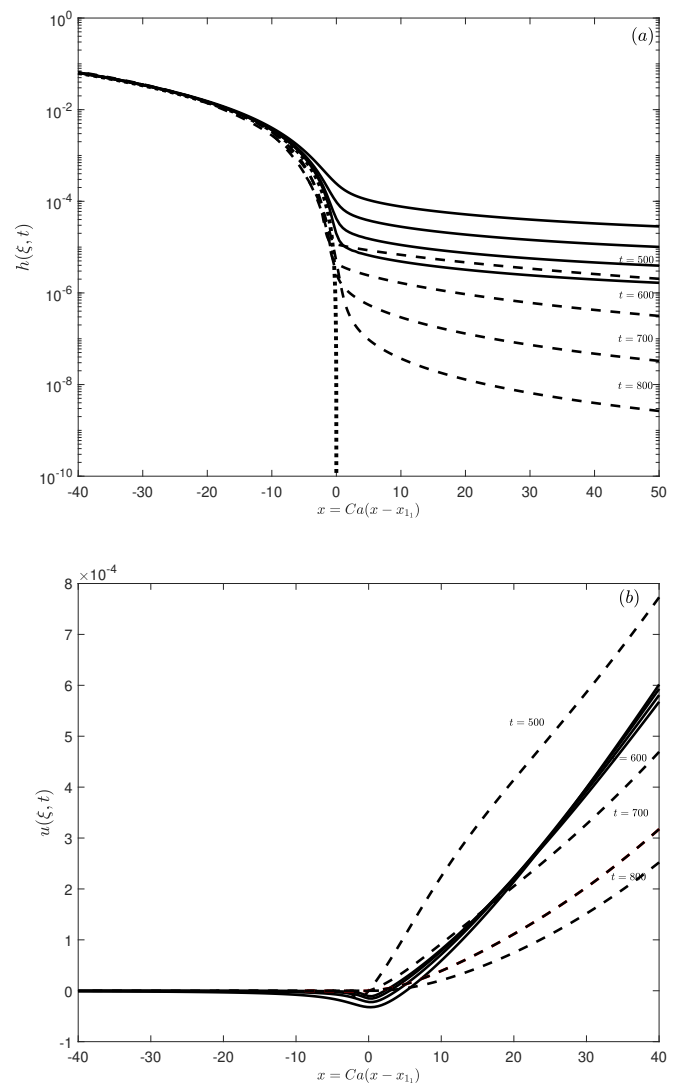

FIG. 24: Computed film thickness profiles, $h,(a)$ plotted on a semi-logarithmic axis, and the extensional flow speed, $u,(b)$ (solid lines) using the similarity coordinate $\xi=C a\left(x-x_{1_{1}}(t)\right)$ for $t=(5,6,7,8) \times 10^{2}$.

The dashed lines show the corresponding late-time similarity solution given by Eqs. (B58,B61). The dotted curve in $(a)$ shows the quasi-static solution of region I given by Eq. (B20) in the similarity variable $\xi$ for $-C_{a x} \leq \xi \leq 0$. The parameter values are $C a=10^{3}$, $R e=0$ and $x_{1_{\infty}}=0.13464$. of $x_{1}$ computed from the numerical solution shown in Fig. $19(a)$ at the given times, and $x_{1_{1}}(t)=x_{1_{\infty}}=0.13464$. We observe that the similarity solution clearly captures the qualitative characteristics of the quasi-static evolution, namely, the family of solutions of $h$ (see solid lines (numerical solution) and dashed lines (similarity solution) in Fig. 24(a)) emanating from the quasi-static solution of region I given by Eq. (B20) in the similarity variable $\xi$ for $-\operatorname{Cax}_{1_{1}} \leq \xi \leq 0$ (see dotted line in Fig. $24(a)$ ), and the reversal of the flow $u$ near $\xi=0$ (Fig. $24(b))$. Although the quantitative match is not perfect, the similarity solution given by Eqs. (B57,B55) capture very well the dynamics in this transition region.

\section{a. Closure relationships to determine evolution of characteristic variables}

The solution of the boundary value problems given by Eqs. (B57,B61) depend on the values of $\mathrm{Ca}$ and the characteristic quantities, $x_{1,2}$ and $Q_{1,2}$, at any instant of time starting from some initial conditions $x_{1,2}^{(0)}$. Note that $x_{\{1,2\}_{1}}(t)$ is almost constant (see Fig. $19(a)$ ), and we assume that $x_{1_{1}}(t)=x_{1_{\infty}}=0.13464$ and $x_{2_{1}}(t)=x_{2_{\infty}}=$ 0.511. The solution to the boundary value problem in Eq. (B57) provides the flux $Q_{2}$ as a function of $x_{2}$, at any instant of time. Similarly, the solution to the boundary value problem in Eq. (B61) provides the flux $Q_{1}$ as a function of $x_{1}$, at any instant of time. We determine the late-time evolution of $x_{1,2}$ using volume conservation in regions I and II, $\frac{d V_{1}}{d t}=Q_{1}$ and $\frac{d V_{2}}{d t}=Q_{2}$, respectively. The algorithm to obtain the time evolution of $x_{1,2}$, and the corresponding family of solutions of $(\mathrm{B} 57, \mathrm{~B} 61)$ is as follows:

1. Start with initial conditions $x_{1,2}^{(0)}$ at some time $t=$ $t^{(0)}$. Compute the corresponding volume $V_{1}^{(0)}=$ $\int_{0}^{x_{1}^{(0)}} h d x$ and $V_{2}^{(0)}=\int_{x_{2}^{(0)}}^{1} h d x$.

2. Solve the boundary value problems in Eqs. $(\mathrm{B} 57, \mathrm{~B} 61)$ to obtain the initial flux, $Q_{1,2}^{(0)}$.

3. Determine the values of $x_{1,2}^{(1)}$ at some time $t=$ $t^{(0)}+\Delta t$ (where $\Delta t$ is a small increment in time), using the updated volume $V_{1}^{(1)} \approx V_{1}^{(0)}+Q_{1}^{(0)} \Delta t=$ $\int_{0}^{x_{1}^{(1)}} h d x$ and $V_{2}^{(1)} \approx V_{2}^{(0)}+Q_{2}^{(0)} \Delta t=\int_{x_{2}^{(1)}}^{1} h d x$. Using the expressions for $h$ in region I (given by Eq. (B20)) and that in region II (given by Eq. (B53)), these are quartic equations in $x_{1,2}^{(1)}$ which need to be solved to get the updated values of $x_{1,2}$ (similar to Eqs. (B21,B54) for $x_{\{1,2\}_{\infty}}$, respectively).

4. Use $x_{1,2}^{(1)}$ to solve the boundary value problem in Eqs. (B57,B61) to obtain the flux, $Q_{1,2}^{(1)}$ at time $t^{(1)}$. 
5. Now repeat steps 3 and 4 to obtain the solution and characteristic variables at time $t^{(n)}=t^{(0)}+n \Delta t$.

6. Use Eq. (B45) to numerically solve for $h_{\min }$, e.g., using a forward Euler time-stepping scheme, $h_{\text {min }}\left(t^{(n)}\right)=h_{m i n}\left(t^{(n-1)}\right)+\Delta t \frac{\left[Q_{1}^{(n-1)}(t)-Q_{2}^{(n-1)}(t)\right]}{\left[x_{2}^{(n-1)}(t)-x_{1}^{(n-1)}(t)\right]}$.

7. Plot the characteristic variables and the family of solutions as a function of time.

${ }^{1}$ J. Banhart, "Metal foams: Production and stability," Advanced Engineering Materials 8, 781-794 (2006).

${ }^{2}$ M. Scheffler and P. C. (Eds), eds., "Cellular ceramics: Structure, manufacturing, properties and applications," (Wiley, 2005) Chap. 1.2.

${ }^{3}$ D. Weaire, S. Hutzler, S. Cox, N. Kern, M. Alonso, and W. Drenckhan, "The fluid dynamics of foams," J. of Physics: Condensed matter 15, S65-S73 (2003).

${ }^{4}$ A. Koponen, O. Timofeev, A. Jäsberg, and H. Kiiskinen, "Drainage of high-consistency fiber-laden aqueous foams," Cellulose 27, 9637-9652 (2020).

${ }^{5}$ B. Haffner, F. Dunne, S. Burke, and S. Hutzler, "Ageing of fibre-laden aqueous foams," Cellulose 24, 231-239 (2017).

${ }^{6}$ G. Ashby, Cellular Solids: Structure and Properties (Cambridge University Press, 1999).

${ }^{7}$ M. Ashby, A. Evans, N. Fleck, L. Gibson, J. Hutchinson, and $\mathrm{H}$. Wadley, Metal foams: A design guide (ButterworthHeinemann, 2000).

${ }^{8}$ S. Wang, P. Austin, and S. Chakrabati-Bell, "It's a maze: The pore structure of bread crumbs," Journal of Cereal Science 54, 203-210 (2011).

${ }^{9}$ P. Stevenson, Foam Engineering: Fundamentals and Applications (Wiley, 2012).

${ }^{10}$ I. Cantat, S. Cohen-Addad, F. Elias, F. Graner, R. Hohler, O. Pitois, and A. Rouyer, F. Saint-Jalmes, Foams - Structure and Dynamics (Oxford University Press, 2013).

${ }^{11}$ P. A. Netti, ed., Biomedical Foams for Tissue Engineering Applications (Woodhead Publishing, 2014).

${ }^{12} \mathrm{C}$. C. Yang and H. Nakae, "The effects of viscosity and cooling conditions on the foamability of aluminum alloy," J. Mat. Proc. Tech. 141, 202-206 (2003).

${ }^{13}$ M. Safouane, A. Saint-Jalmes, V. Bergeron, and D. Langevin, "Viscosity effects in foam drainage: Newtonian and nonnewtonian foaming fluids," Eur. Phy. J. E 19, 195-202 (2006).

${ }^{14}$ M. Safouane, M. Durand, A. Saint-Jalmes, D. Langevin, and V. Bergeron, "Aqueous foam drainage. role of the rheology of the foaming fluid," J. Phys. IV (France) 11, Pr6 -275 - PR6 $-280(2001)$.
${ }^{15}$ K. J. Mysels, K. Shinoda, and S. Frankel, Soap Films: Studies of Their Thinning (Pergamon, 1959).

${ }^{16} \mathrm{~L}$. Schwartz and R. Roy, "Modeling draining flow in mobile and immobile soap films," J. Colloid and interface Science 218, 309323 (1999).

${ }^{17}$ L. Champougny, E. Rio, F. Restagno, and B. Scheid, "The break-up of free films pulled out of a pure liquid bath," J. Fluid Mechanics 811, 499-524 (2016).

${ }^{8} \mathrm{~S}$. Naire, R. Braun, and S. Snow, "Limiting cases of gravitational drainage of a vertical free film for evaluating surfactants," SIAM J. Applied Maths. 61, 889-913 (2000).

${ }^{19}$ S. Naire, R. Braun, and S. Snow, "An insoluble surfactant model for a vertical draining free film," Journal of Colloid Interface Science 230, 91-106 (2000).

${ }^{20} \mathrm{~S}$. Naire, R. Braun, and S. Snow, "An insoluble surfactant model for a vertical draining free film with variable surface viscosity," Physics of Fluids 13, 2492-2502 (2001).

${ }^{21}$ R. Braun, S. Snow, and S. Naire, "Models for gravitationallydriven free-film drainage," Journal of Engineering Maths. 43, 281-314 (2002).

${ }^{22}$ T. Erneux and S. Davis, "Nonlinear rupture of free films," Physics Fluids 5, 1117-1122 (1993).

${ }^{23} \mathrm{C}$. Breward, The mathematics of foam, Ph.D. thesis, Oxford University (1999).

${ }^{24}$ C. Breward and P. Howell, "The drainage of a foam lamella," Journal Fluid Mechanics 458, 379-406 (2002).

${ }^{25} \mathrm{~L}$. Brush and S. Davis, "A new law of thinning in foam dynamics," Journal Fluid Mechanics 534, 227-236 (2005).

${ }^{26} \mathrm{~L}$. Schwartz and H. Princen, "A theory of extensional viscosity for flowing foams and concentrated emulsions," J. Colloid Interface Science 118, 201-211 (1987).

${ }^{27}$ M. Davis, P. Stewart, and S. Davis, "Local effects of gravity on foams," J. Fluid Mechanics 737, 1-18 (2013).

${ }^{28} \mathrm{~L}$. Brush and S. Roper, "The thinning of lamellae in surfactantfree foams with non-newtonian liquid phase," Journal Fluid Mechanics 616, 235-262 (2008).

${ }^{29}$ T. G. Myers, "Application of non-newtonian models to thin film flow," Physical Review E 6, 066302-066302 (2005).

${ }^{30}$ N. J. Balmforth, A. S. Burbridge, R. V. Craster, J. Salzig, and A. Shen, "Visco-plastic models of isothermal lava domes," Journal of Fluid Mechanics 403 (2000).

${ }^{31}$ G. D. Smith, Numerical Solution of Partial Differential Equations: Finite Difference Methods, third edition ed. (Macmillan, 1985).

${ }^{32}$ A. Alhushaybari and J. Uddin, "Convective and absolute instability of viscoelastic liquid jets in the presence of gravity," Physics of Fluids 6, 044106 (2019).

${ }^{33} \mathrm{~A}$. Alhushaybari and J. Uddin, "Absolute instability of freefalling viscoelastic liquid jets with surfactants," Physics of Fluids 32, 013102 (2020).

${ }^{34} \mathrm{P}$. Stewart and S. Davis, "Dynamics and stability of metallic foams: network modelling," Journal of Rheology 56, 543-574 (2012). 